\title{
A new statistical test based on the wavelet cross-spectrum to detect time-frequency dependence between non-stationary signals: application to the analysis of cortico-muscular interactions
}

\author{
Jérémie Bigot ${ }^{1,2}$, Marieke Longcamp ${ }^{3,5}$, Fabien Dal Maso ${ }^{3}$ \& David Amarantini ${ }^{3,4}$ \\ Institut de Mathématiques de Toulouse et CNRS (UMR 5219) ${ }^{1}$, Université de Toulouse \\ Center for Mathematical Modelling², Universidad de Chile \\ $\&$ \\ LAPMA $^{3}$, Université de Toulouse \\ Département de kinésiologie ${ }^{4}$, Centre de réadaptation Marie-Enfant, Université de Montréal \\ INCM (UMR 6193)5 ${ }^{5}$ CNRS-Université de la Méditerranée, Marseille, France \\ January 13, 2011
}

\begin{abstract}
The study of the correlations that may exist between neurophysiological signals is at the heart of modern techniques for data analysis in neuroscience. Wavelet coherence is a popular method to construct a time-frequency map that can be used to analyze the time-frequency correlations between two time series. Coherence is a normalized measure of dependence, for which it is possible to construct confidence intervals, and that is commonly considered as being more interpretable than the wavelet cross-spectrum (WCS). In this paper, we provide empirical and theoretical arguments to show that a significant level of wavelet coherence does not necessarily correspond to a significant level of dependence between random signals, especially when the number of trials is small. In such cases, we demonstrate that the WCS is a much better measure of statistical dependence, and a new statistical test to detect significant values of the cross-spectrum is proposed. This test clearly outperforms the limitations of coherence analysis while still allowing a consistent estimation of the time-frequency correlations between two non-stationary stochastic processes. Simulated data are used to investigate the advantages of this new approach over coherence analysis. The method is also applied to experimental data sets to analyze the time-frequency correlations that may exist between electroencephalogram (EEG) and surface electromyogram (EMG).
\end{abstract}

Keywords: Coherence; Cross-specturm; Wavelet; Time-frequency dependence; Statistical testing; Corticomuscular interactions

2010 MSC classifications: 62M10, 62P10

\section{Introduction}

In the last decades, the oscillatory behavior of neurophysiological signals has drawn increased attention among neuroscientists BD04, SH03, SG05, VLRM01. In this framework, the correlations occurring at different frequencies between two or more signals are assumed to indicate oscillatory coupling of neuronal groups. Typically, neurophysiological signals contain noise at different frequency 
bands and are thus considered as random time series or stochastic processes. Fourier analysis has been widely used for studying the spectral contents of such signals, and the correlations that may exist at different frequencies between electroencephalogram (EEG), electromyogram (EMG) and magnetoencephalogram (MEG) GCB02, HCFR98, HRA ${ }^{+95}$, MH99b, MSGH00, have been investigated using Fourier coherence. If the investigated signals are assumed to be stationary, then estimates of the auto and cross-spectra can be calculated to compute an estimation of the coherence at different frequencies. However, signals typically encountered in biomedical applications are non-stationary times series whose frequency behavior changes with time. A powerful alternative to Fourier analysis is the wavelet transform which is a widely developed tool for the study of non-stationary signals as it allows a simultaneous analysis of the content of a signal over time and frequency (see e.g. Mal98. for an introduction to wavelet analysis, and AM10 for a review and comparison of time-frequency methods for the analysis of EEG signals). In recent years, wavelet coherence has been proposed as an alternative to Fourier coherence for the analysis of time-frequency dependence between two time series. There exists a rich literature on estimating time-frequency dependence between time series using localized transforms, see e.g. CAvSRA01, SFJ10, WCB05, Whi00. Applications of time-frequency coherence can be found in neuroscience $\mathrm{LLR}^{+} 02$, OVB08, $\mathrm{ZHJ}^{+} 06$, but also in geophysics GMJ04, MK04, and wind engineering GKK03 to name but a few. The wavelet coherence is a normalized measure (between 0 and 1) of time-frequency dependence between two time series that is commonly considered as being more interpretable than the wavelet cross-spectrum (WCS). Statistical tests have been proposed in GC88, HRA ${ }^{+}$95, MSF07] to derive confidence intervals for the Fourier coherence between two-time series. A similar statistical test, based on averaging over repeated trials, has been suggested in $\left[\mathrm{ZHJ}^{+} 06\right.$ to determine if the time-frequency coherence between neurophysiological signals is significant or not.

The objective of this paper is to discuss the relevance of wavelet coherence as a statistical measure of time-frequency dependence between random time series, and to compare it to the statistical information contained in the WCS. The main contributions are the following: first theoretical and empirical arguments are detailed to show some limitations of wavelet coherence analysis. In particular, we exhibit some drawbacks of the test proposed in $\left[\mathrm{ZHJ}^{+} 06\right]$ which may yield an erroneous estimation of time-frequency correlations between two signals.

Second, as an alternative to the limitations of wavelet coherence, a new statistical test to detect significant values of the WCS is proposed. Contrary to the standard test in $\mathrm{GC}^{2}, \mathrm{HRA}^{+} 95, \mathrm{ZHJ}^{+} 06$. to derive confidence intervals for the coherence, our test correctly estimates the areas in the timefrequency plane where the dependence between the time series is truly significant, and does not detect any area where no correlation between the signals exists (an example being the case of two independent Gaussian time series with zero mean). The idea of the test is rather simple. High values of the WCS should correspond to areas in the time-frequency plane where there exists a correlation between the time series. Thus, we first derive a threshold such that, with high probability and under the null hypothesis that the observed signals are independent Gaussian times series, all the values of the WCS fall below this threshold. This means that the values of the WCS that do not correspond to a significant level of time-frequency dependence lie below a certain value with large probability. We show that this probabilistic bound is a threshold that can be easily computed from the data. A standard criticism on the use of the cross-spectrum is that one cannot assess the strength of dependence because this measure does not take into account the variances of the time series contrary to the coherence. To overcome this issue, our method automatically includes an estimation of the variance of the two time series in the computation of the threshold. Therefore, the procedure is fully data-driven, and it is in particular adapted to the case where one pair of time series has a higher 
magnitude of covariance that the second pair. This new test is also based on averaging over repeated trials as in $\mathrm{ZHJ}^{+} 06$. However, the proposed probabilistic bound is non-asymptotic, meaning that it holds for any values of the number of trials and length of the signals. Moreover, the test is valid for any Gaussian processes with zero-mean without assuming stationarity or making parametric assumptions on the covariance functions of the time series. It is therefore very robust in the sense that excellent results can be obtained for a broad class of random signals using very few trials.

Third, a detailed simulation study is proposed to investigate the advantages of this new approach over coherence analysis, and the method is applied to experimental data sets to analyze the timefrequency correlations that may exist between EEG and EMG signals.

\section{Methods}

\subsection{The standard test for detecting significant values of wavelet coherence}

First, let us fix the notations and recall the definitions of wavelet transform, WCS and wavelet coherence between stochastic processes. Let $\mathbf{x}=\left[x\left(t_{k}\right)\right]_{k=1}^{T}$ and $\mathbf{y}=\left[y\left(t_{k}\right)\right]_{k=1}^{T}$ denote two random time series of length $T$ observed at regularly spaced time points $t_{k}$. The wavelet transform (WT) of $\mathbf{x}$ (resp. y) at scale $s>0$ and time $u$ is defined as (see e.g. Mal98)

$$
W_{\mathbf{X}}(s, u)=\sum_{k=1}^{T} x\left(t_{k}\right) \overline{\psi_{s, u}\left(t_{k}\right)}
$$

where $\bar{z}$ denotes the conjugate of a complex number $z$,

$$
\psi_{s, u}\left(t_{k}\right)=\frac{1}{\sqrt{s}} \psi\left(\frac{t_{k}-u}{s}\right)
$$

and $\psi(\cdot)$ is an oscillating function called wavelet which should satisfy a number of regularity and admissibility conditions (see e.g. Mal98]). The WT can be seen as a time-frequency representation of a signal by converting the scale parameter $s$ to a frequency parameter $\omega$. This correspondence depends on a specific frequency $\omega_{0}$, which represents the central frequency location of the energy $\psi$ in the Fourier domain, and the relationship between frequency and scale is given by $\omega \approx \frac{\omega_{0}}{s}$. Thus the WT at frequency $\omega$ and time $u$ can be expressed as

$$
W_{\mathbf{X}}(\omega, u)=\sum_{k=1}^{T} x\left(t_{k}\right) \sqrt{\frac{\omega}{\omega_{0}}} \psi\left(\frac{\omega}{\omega_{0}}\left(t_{k}-u\right)\right) .
$$

A commonly used wavelet in practice is the Morlet wavelet, which is a complex-valued function, defined as

$$
\psi(u)=\pi^{-1 / 4} e^{i \omega_{0} u} e^{-u^{2} / 2},
$$

where $\omega_{0}$ is the central frequency of $\psi$. A Morlet wavelet is thus a complex sine wave within a Gaussian envelope, and the parameter $\omega_{0}$ determines the number of oscillations of the wavelet within this envelope. In all the numerical experiments presented in this paper, we took $\omega_{0}=7$ as it is a common choice in wavelet analysis of neurophysiological signals, see e.g. [TBBDP96]. 
The wavelet coherence at frequency $\omega$ and time $u$ between the time series $\mathbf{x}$ and $\mathbf{y}$ is then defined by (see e.g. GMJ04, MK04, ZHJ ${ }^{+} 06$ ])

$$
R_{\mathbf{x y}}^{2}(\omega, u)=\frac{\left|S_{\mathbf{x y}}(\omega, u)\right|^{2}}{S_{\mathbf{x}}(\omega, u) S_{\mathbf{y}}(\omega, u)}
$$

where $S_{\mathbf{x y}}(\omega, u)$ is the WCS between $\mathbf{x}$ and $\mathbf{y}$, and $S_{\mathbf{X}}(\omega, u)$ (resp. $S_{\mathbf{y}}(\omega, u)$ ) is the wavelet autospectrum (WAS) of $\mathbf{x}$ (resp. $\mathbf{y}$ ) defined respectively as

$$
S_{\mathbf{X y}}(\omega, u)=\mathbb{E}\left(W_{\mathbf{X}}(\omega, u) \overline{W_{\mathbf{y}}(\omega, u)}\right) \text { and } S_{\mathbf{X}}(\omega, u)=\mathbb{E}\left|W_{\mathbf{X}}(\omega, u)\right|^{2},
$$

where $\mathbb{E} Z$ denotes the expectation of a random variable $Z$.

Recall that if one observes $n$ independent realizations $Z_{m}, m=1, \ldots, n$ of $Z$ then $\mathbb{E} Z=$ $\lim _{n \rightarrow+\infty} \frac{1}{n} \sum_{m=1}^{n} Z_{m}$ by the law of large numbers. Hence, if one observes data consisting of $n$ repeated trials $\left(\mathbf{x}_{m}\right)_{m=1, \ldots, n}=\left(\left[x_{m}\left(t_{k}\right)\right]_{k=1}^{T}\right)_{m=1, \ldots, n}$ and $\left(\mathbf{y}_{m}\right)_{m=1, \ldots, n}=\left(\left[y_{m}\left(t_{k}\right)\right]_{k=1}^{T}\right)_{m=1, \ldots, n}($ viewed as $n$ independent realizations of the stochastic processes $\mathbf{x}$ and $\mathbf{y}$ respectively) then the WCS between the two time series is naturally estimated by the following empirical wavelet cross-spectrum

$$
\hat{S}_{\mathbf{x y}}(\omega, u)=\frac{1}{n} \sum_{m=1}^{n} W_{\mathbf{x}_{m}}(\omega, u) \overline{W_{\mathbf{y}_{m}}(\omega, u)}
$$

and an estimator of the wavelet coherence is given by the following empirical wavelet coherence

$$
\hat{R}_{\mathbf{x y}}^{2}(\omega, u)=\frac{\left|\sum_{m=1}^{n} W_{\mathbf{x}_{m}}(\omega, u) \overline{W_{\mathbf{y}_{m}}(\omega, u)}\right|^{2}}{\left(\sum_{m=1}^{n}\left|W_{\mathbf{x}_{m}}(\omega, u)\right|^{2}\right)\left(\sum_{m=1}^{n}\left|W_{\mathbf{y}_{m}}(\omega, u)\right|^{2}\right)} .
$$

Note that the "true" coherence $R_{\mathbf{x y}}^{2}(\omega, u)$ is the limit as the number of trials tends to infinity $(n \rightarrow+\infty)$ of the above empirical coherence $\hat{R}_{\mathbf{x y}}^{2}(\omega, u)$.

The wavelet coherence is a normalized measure (between 0 and 1) of time-frequency dependence between two time series. Note that if $\mathbf{x}$ and $\mathbf{y}$ are independent zero-mean processes then, at any frequency $\omega$ and time $u$, one has that

$$
S_{\mathbf{X y}}(\omega, u)=\mathbb{E}\left(W_{\mathbf{X}}(\omega, u)\right) \mathbb{E}\left(\overline{W_{\mathbf{y}}(\omega, u)}\right)=0
$$

and thus $R_{\mathbf{x y}}^{2}(\omega, u)=0$. To the contrary, if there exists a linear relationship $W_{\mathbf{y}}(\omega, u)=a W_{\mathbf{x}}(\omega, u)$ between $\mathbf{x}$ and $\mathbf{y}$ at some frequency $\omega$ and time $u$ with $a \neq 0$, then $S_{\mathbf{x y}}(\omega, u)=\bar{a} S_{\mathbf{X}}(\omega, u)$ and $S_{\mathbf{y}}(\omega, u)=|a|^{2} S_{\mathbf{X}}(\omega, u)$ which implies that $R_{\mathbf{x y}}^{2}(\omega, u)=1$. Therefore, values of wavelet coherence close to 1 are interpreted as evidence for a significant time-frequency correlation between $\mathbf{x}$ and $\mathbf{y}$. In practice, as a preliminary step, the observed times series can be centered to have zero mean over the $n$ repetitions before computing the empirical wavelet cross-spectrum and coherence.

To derive a threshold to detect automatically significant values of the coherence, most authors in the literature use a procedure proposed in GC88 to test the null hypothesis $H_{0}$ that the two time series $\mathbf{x}$ and $\mathbf{y}$ are independent Gaussian white noise. Based on repeated observations $\left(\mathbf{x}_{m}, \mathbf{y}_{m}\right)_{m=1, \ldots, n}$ with $n \geq 2$, it has been shown in GC88 that, under $H_{0}, \hat{R}_{\mathbf{x y}}^{2}(\omega, u) \leq r_{\alpha}$ with probability $1-\alpha$ where the threshold $r_{\alpha}$ is equal to

$$
r_{\alpha}=1-\alpha^{1 /(n-1)} \text { for } 0 \leq \alpha \leq 1 .
$$

Therefore, at level $\alpha=5 \%$, a detection threshold is $r_{\alpha}=1-0.05^{1 /(n-1)}$ and values of $\hat{R}_{\mathbf{x y}}^{2}(\omega, u)$ that are above this level are considered as a significant level of coherence. 


\subsection{A new statistical test for detecting significant values of the wavelet cross- spectrum}

It is often argued that wavelet coherence is more interpretable than the WCS which is a nonnormalized measure of dependence. Indeed, at first glance, it seems difficult to judge if an observed value of the cross-spectrum is significant. Let us recall that the standard method to detect significant values of the coherence is based on a statistical procedure to test the null hypothesis $H_{0}$ that the components $x\left(t_{k}\right)$ and $y\left(t_{k}\right)$ of the two time series $\mathbf{x}$ and $\mathbf{y}$ are independent and identically distributed (iid) centered Gaussian variables (that is $x\left(t_{k}\right) \sim_{i i d} N\left(0, \sigma_{x}^{2}\right)$ and $y\left(t_{k}\right) \sim_{i i d} N\left(0, \sigma_{y}^{2}\right)$ for $\left.k=1, \ldots, T\right)$. We propose to derive a new statistical procedure to test the more general null hypothesis $H_{0}\left(\Sigma_{x}, \Sigma_{y}\right)$ that the random time series $\mathbf{x}=\left[x\left(t_{k}\right)\right]_{k=1}^{T}$ and $\mathbf{y}=\left[y\left(t_{k}\right)\right]_{k=1}^{T}$ of length $T$ are independent Gaussian vectors with zero mean and covariance matrix $\Sigma_{x}$ and $\Sigma_{y}$ respectively, namely

$$
\begin{aligned}
H_{0}\left(\Sigma_{x}, \Sigma_{y}\right): & \mathbf{x} \text { and } \mathbf{y} \text { are independent vectors of length } T \text { with } \\
& \mathbf{x} \sim N\left(0, \Sigma_{x}\right) \text { and } \mathbf{y} \sim N\left(0, \Sigma_{y}\right),
\end{aligned}
$$

where $N(0, \Sigma)$ denotes a Gaussian random vector of length $T$ with zero mean and covariance matrix $\Sigma$. Note that $H_{0}\left(\Sigma_{x}, \Sigma_{y}\right)$ includes the case where the components of the two time series $\mathbf{x}$ and $\mathbf{y}$ are iid centered Gaussian variables with variance $\sigma_{x}^{2}$ and $\sigma_{y}^{2}$ which corresponds to the choice $\Sigma_{x}=\sigma_{x}^{2} I_{T}$ and $\Sigma_{y}=\sigma_{y}^{2} I_{T}$ where $I_{T}$ denotes the identity matrix of size $T \times T$.

Theoretical arguments developed in the Appendix show that under $H_{0}\left(\Sigma_{x}, \Sigma_{y}\right),\left|\hat{S}_{\mathbf{x y}}(\omega, u)\right| \leq \hat{\lambda}_{\alpha}$ with probability larger than $1-\alpha$ where the threshold $\hat{\lambda}_{\alpha}$ is equal to

$$
\hat{\lambda}_{\alpha}=\frac{\hat{\rho}_{x} \hat{\rho}_{y}}{\left(1+\sqrt{\frac{T}{n}}\right)^{2}}\left(-\frac{\log (\alpha / 2)}{n}+\sqrt{-\frac{2 \log (\alpha / 2)}{n}}\right),
$$

with $\hat{\rho}_{x}^{2}$ (resp. $\hat{\rho}_{y}^{2}$ ) being the largest eigenvalue of the empirical covariance matrix of the time serie $\mathbf{x}$ (resp. y). We refer the Appendix for a precise definition, and for the computation of $\hat{\lambda}_{\alpha}$ in practice. Using such a procedure, the values of the empirical cross-spectrum that are lower than the threshold $\hat{\lambda}_{\alpha}$ (with e.g. $\alpha=5 \%$ ) are considered as not significant, whereas the values $\left|\hat{S}_{\mathbf{x y}}(\omega, u)\right|$ that are above the threshold $\hat{\lambda}_{\alpha}$ can be considered as being a truly significant level of time-frequency dependence at frequency $\omega$ and time $u$. Note that this test does not make any parametric assumption on the covariance matrices $\Sigma_{x}$ and $\Sigma_{y}$.

As explained previously, coherence is a normalized measure of dependence that is usually considered as being more interpretable that the cross-spectrum which does not take into account the variance of the time series to assess the strength of dependence. Hence, if one pair of time series has a very large auto-spectrum this may cause the cross-spectrum to be very large even in the absence of dependence between two time series. In the computation of the threshold $\hat{\lambda}_{\alpha}$, the term $\frac{\hat{\rho}_{x} \hat{\rho}_{y}}{\left(1+\sqrt{\frac{T}{n}}\right)^{2}}$ corresponds to a data-based upper bound of the amplitude of the auto-spectra of the time series.

Indeed, consider first the null hypothesis $H_{0}\left(\sigma_{x}^{2} I_{T}, \sigma_{y}^{2} I_{T}\right)$. Under such an assumption, the test is based on the properties that

- with probability larger than 1- $\alpha:\left|\hat{S}_{\mathbf{x y}}(\omega, u)\right|^{2} \leq \sigma_{x}^{2} \sigma_{y}^{2} * C(n, \alpha)$ where $C(n, \alpha)=\left(-\frac{\log (\alpha / 2)}{n}+\sqrt{-\frac{2 \log (\alpha / 2)}{n}}\right)^{2}$ is a constant depending only on $n$ and the level of the test $\alpha$. 
- with large probability: $\hat{\lambda}_{\alpha}^{2} \approx \sigma_{x}^{2} \sigma_{y}^{2} * C(n, \alpha)$.

Hence, the computation of the threshold $\hat{\lambda}_{\alpha}$ includes an estimation of the variance $\sigma_{x}^{2}$ and $\sigma_{y}^{2}$ of each time serie.

In the more general case where $\Sigma_{x} \neq \sigma_{x}^{2} I_{T}$ or $\Sigma_{y} \neq \sigma_{y}^{2} I_{T}$, the test uses the properties that

- with probability larger than $1-\alpha:\left|\hat{S}_{\mathbf{x y}}(\omega, u)\right|^{2} \leq S_{\mathbf{X}}(\omega, u) S_{\mathbf{y}}(\omega, u) * C(n, \alpha)$ where $S_{\mathbf{X}}(\omega, u)$ (resp. $\left.S_{\mathbf{y}}(\omega, u)\right)$ is the wavelet auto-spectrum of $\mathbf{x}$ (resp. $\left.\mathbf{y}\right)$.

- with large probability: $\hat{\lambda}_{\alpha}^{2} \geq S_{\mathbf{X}}(\omega, u) S_{\mathbf{y}}(\omega, u) * C(n, \alpha)$, which corresponds to a data-based upper bound for the product of the wavelet auto-spectra of the two time series.

Thus, the above arguments show that the test automatically adapts to the case where one pair of time serie has a large wavelet auto-spectra (which results in a large wavelet cross-spectrum) while still controlling the level of dependence between the two time series. Such a test is also non-asymptotic in the sense that it holds for any value of number of trials $n$ and length of the signals $T$.

\section{$3 \quad$ Results}

\subsection{Simulated data}

Let us first consider some simulated examples to illustrate the differences between the test based on wavelet coherence (using the threshold $r_{\alpha}$ ), and the test based on the WCS (using the data-based threshold $\hat{\lambda}_{\alpha}$ ) for the detection of time-frequency correlations between random signals.

\subsubsection{Analysis of time-frequency dependent Gaussian processes}

Example 1 - We simulate $n$ independent realizations (trials) of the following two Gaussian times series with zero mean of length $T=1000 \mathrm{~ms}$ generated with sampling rate $1 \mathrm{kHz}$ :

$$
\begin{aligned}
x\left(t_{k}\right) & =Z\left(\sin \left(2 \pi \omega_{1} t_{k}\right) \mathbb{1}_{\left[0, u_{1}[\right.}\left(t_{k}\right)+\sin \left(2 \pi \omega_{2} t_{k}\right) \mathbb{1}_{\left[u_{1}, u_{2}[\right.}\left(t_{k}\right)\right)+\sigma_{x} \epsilon_{1, k} \\
y\left(t_{k}\right) & =Z\left(a_{1} \sin \left(2 \pi \omega_{1} t_{k}\right) \mathbb{1}_{\left[0, u_{1}[\right.}\left(t_{k}\right)+a_{2} \sin \left(2 \pi \omega_{2} t_{k}\right) \mathbb{1}_{\left[u_{1}, u_{2}[\right.}\left(t_{k}\right)\right)+\sigma_{y} \epsilon_{2, k}, \\
\text { where } Z & \sim N(0,1)
\end{aligned}
$$

with $t_{k}=1, \ldots, T, \omega_{1}=10 \mathrm{~Hz}, \omega_{2}=30 \mathrm{~Hz}, u_{1}=300 \mathrm{~ms}, u_{2}=700 \mathrm{~ms}, a_{1}=1.2, a_{2}=1.5$, and where the $\epsilon_{j, k}$ 's are independent Gaussian variables with zero mean and variance 1 . The parameters $\sigma_{x}, \sigma_{y}$ are levels of noise that can be adjusted according to the desired signal-to-noise ratio (SNR). For two reals $a$ and $b, \mathbb{1}_{[a, b[}\left[t_{k}\right)$ denotes the function which is equal to 0 if $t_{k}<a$ or $t_{k} \geq b$ and to 1 otherwise. Note that for the signal $\mathbf{x}$, the SNR is defined as $20 \log _{10}\left(1 / \sigma_{x}\right)$, and for the signal $\mathbf{y}$, the SNR is $20 \log _{10}\left(a_{2} / \sigma_{y}\right)$. The two times series are thus two sine waves with random amplitude of different frequency and time localization with additive Gaussian white noise. It should be noted that the amplitude of the two time series are correlated on the time intervals $[0,300 \mathrm{~ms}]$ and $[300 \mathrm{~ms}, 700 \mathrm{~ms}]$ at frequency $\omega_{1}=10 \mathrm{~Hz}$ and $\omega_{2}=30 \mathrm{~Hz}$ respectively. Thus $\mathbf{x}$ and $\mathbf{y}$ are time-frequency dependent Gaussian processes.

An example of realization for each time serie with a SNR equal to $-5 \mathrm{~dB}$ is given in Figure 1(a). The wavelet coherence $R_{\mathbf{x y}}^{2}(\omega, u)$ and the "true" WCS $S_{\mathbf{x y}}(\omega, u)$ are displayed in Figure $1(\mathrm{~d}, \mathrm{e})$. The empirical coherence $\hat{R}_{\mathbf{x y}}^{2}(\omega, u)$ computed with $n=10$ trials is displayed in Figure 2(c) together 
with a time-frequency map showing (in red) the values of the empirical coherence that are above the threshold $r_{\alpha}$ with $\alpha=5 \%$.

First, remark the wavelet coherence $R_{\mathbf{x y}}^{2}(\omega, u)$ and the "true" WCS $S_{\mathbf{x y}}(\omega, u)$ do not contain the same information. Large value of the cross-spectrum are mainly observed in narrow frequency bands centered at the frequencies $\omega=10 \mathrm{~Hz}$ and $\omega=30 \mathrm{~Hz}$ and on the time intervals [0,300] $\mathrm{ms}$ and $[300,700] \mathrm{ms}$ which is consistent with model (3.1). To the contrary, the large values of wavelet coherence are much more spread in the time-frequency plane, and are found for example around the point $(\omega, u)=(20 \mathrm{~Hz}, 300 \mathrm{~ms})$ which is somewhat unexpected in the sense that there does not exist such a time-frequency correlation between the two time series in model (3.1).

Secondly, it can be seen from Figure 2(d) that the statistical test using the threshold $r_{\alpha}$ finds significant values for the coherence in the time-frequency plane around the points $(\omega, u)=(10 \mathrm{~Hz}, 200 \mathrm{~ms})$ and $(\omega, u)=(30 \mathrm{~Hz}, 500 \mathrm{~ms})$ which is consistent with model (3.1) used to simulate the data. However, the test also detects areas in the time-frequency plane which do not correspond to significant values of the true coherence displayed in Figure 1(d), or to an expected time-frequency dependence for such data. Now, let us consider the new statistical test suggested in Section 2.2 that is based on the thresholding of the empirical WCS with the data-based threshold $\hat{\lambda}_{\alpha}$. In Figure 2(f) we display the result of this thresholding procedure for data from model (3.1), i.e Example 1 with $n=10$ trials. One can see that the results are much better than those obtained by thresholding the empirical wavelet coherence with $r_{\alpha}$. This new test correctly estimates the areas in the time-frequency plane where the dependence between the time series is truly significant, and does not detect any area where no correlation between the signals exists.
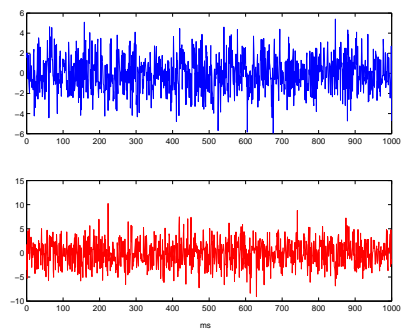

(a)

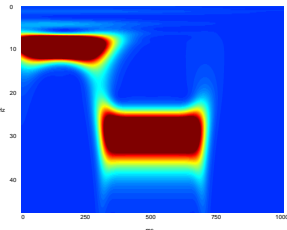

(b)

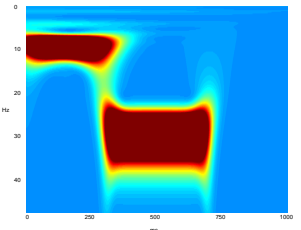

(c)

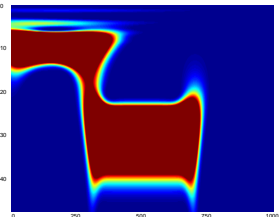

(d)

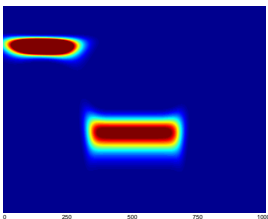

(e)

Figure 1: Example 1 - (a) An example of time series $\mathbf{x}_{1}$ and $\mathbf{y}_{1}$ generated from model (3.1) with SNR $=-5 d B$. (b) Wavelet auto-spectum $S_{\mathbf{X}}(\omega, u)$. (c) Wavelet auto-spectum $S_{\mathbf{y}}(\omega, u)$. (d) Wavelet coherence $R_{\mathbf{x y}}^{2}(\omega, u)$. (e) Wavelet cross-spectrum $\left|S_{\mathbf{x y}}(\omega, u)\right|^{2}$. - The two time series have a common sine wave of $10 \mathrm{~Hz}$ from $1 \mathrm{~ms}$ to $300 \mathrm{~ms}$ and another common sine wave of $30 \mathrm{~Hz}$ from $300 \mathrm{~ms}$ to $700 \mathrm{~ms}$ in each trial.

Results obtained using only $n=2$ trials are displayed in Figure 3. It can be seen that the 


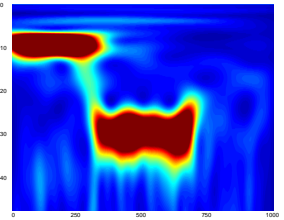

(a)

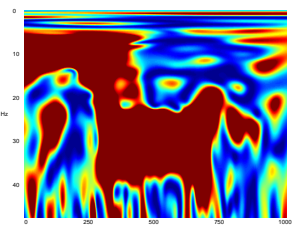

(c)

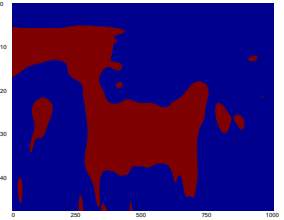

(d)

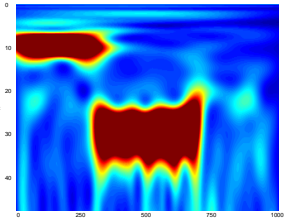

(b)

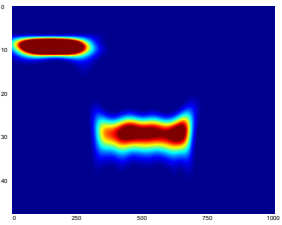

(e)

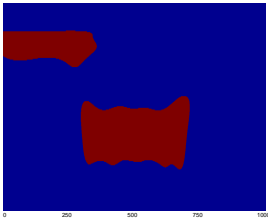

(f)

Figure 2: Example 1 - Automatic detection of time-frequency dependence using $n=10$ trials with $\mathrm{SNR}=-5 d B$. (a) Empirical wavelet auto-spectum $\hat{S}_{\mathbf{X}}(\omega, u)$. (b) Empirical wavelet auto-spectum $\hat{S}_{\mathbf{y}}(\omega, u)$. (c) Empirical wavelet coherence $\hat{R}_{\mathbf{x y}}^{2}(\omega, u)$. (d) Significant values (in red) of the empirical wavelet coherence that are above the threshold $r_{\alpha}$. (e) Empirical WCS $\left|\hat{S}_{\mathbf{x y}}(\omega, u)\right|^{2}$. (f) Significant values (in red) of the empirical WCS that are above the threshold $\hat{\lambda}_{\alpha}$ - The value of $\alpha$ is $5 \%$ for both thresholds.

results using the threshold $r_{\alpha}$ to detect significant values of the wavelet coherence are worse. Indeed, most of the truly significant values of the coherence found previously with $n=10$ trials (around the points $(\omega, u)=(10 \mathrm{~Hz}, 200 \mathrm{~ms})$ and $(\omega, u)=(30 \mathrm{~Hz}, 500 \mathrm{~ms}))$ fall below the threshold $r_{\alpha}$ when using $n=2$ trials. To the contrary, the results displayed in Figure 3(f) show that our procedure using the threshold $\hat{\lambda}_{\alpha}$ to detect significant values of the wavelet cross-spectrum performs very well with only $n=2$ trials.

\subsubsection{Coherence detection in the absence of time-frequency dependence between sig- nals}

Let us now compare the behavior of the two tests when there is a priori no time-frequency dependence between the signals. A simple example being the case of two independent Gaussian time series with zero mean. For this consider the following simulated experiments:

Example 2 - We simulate $n$ independent realizations (trials) of the following two times series of length $T=1000 \mathrm{~ms}$ generated with sampling rate $1 \mathrm{kHz}$ :

$$
\begin{aligned}
& x\left(t_{k}\right)=Z\left(\sin \left(2 \pi \omega_{1} t_{k}\right) \mathbb{1}_{\left[0, u_{1}[\right.}\left(t_{k}\right)+\sin \left(2 \pi \omega_{2} t_{k}\right) \mathbb{1}_{\left[u_{1}, u_{2}[\right.}\left(t_{k}\right)\right)+\sigma_{x} \epsilon_{1, k} \\
& y\left(t_{k}\right)=\sigma_{y} \epsilon_{2, k},
\end{aligned}
$$

where $Z \sim N(0,1)$,

with $t_{k}=1, \ldots, T, \omega_{1}=10 \mathrm{~Hz}, \omega_{2}=30 \mathrm{~Hz}, u_{1}=300 \mathrm{~ms}, u_{2}=700 \mathrm{~ms}$, and where the $\epsilon_{j, k}$ 's are independent Gaussian variables with zero mean and variance 1. The second signal y is therefore a purely Gaussian white noise (we took $\sigma_{y}=\sigma_{x}$ in this numerical example), and therefore the coherence between $\mathbf{x}$ and $\mathbf{y}$ is expected to be zero. An example of coherence estimation using model (3.2) 


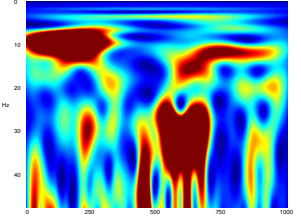

(a)

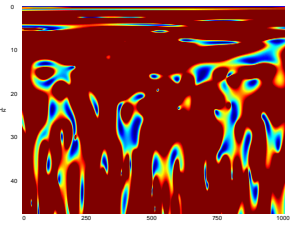

(c)

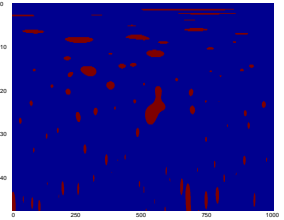

(d)

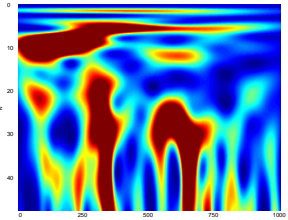

(b)

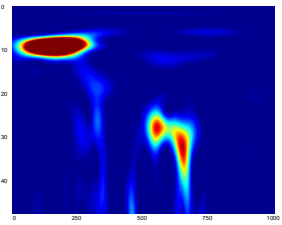

(e)

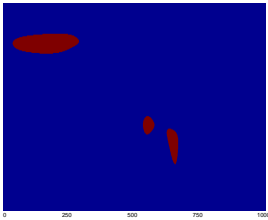

(f)

Figure 3: Example 1 - Automatic detection of time-frequency dependence using $n=2$ trials with SNR $=-5 d B$. (a) Empirical wavelet auto-spectum $\hat{S}_{\mathbf{X}}(\omega, u)$. (b) Empirical wavelet auto-spectum $\hat{S}_{\mathbf{y}}(\omega, u)$. (c) Empirical wavelet coherence $\hat{R}_{\mathbf{x y}}^{2}(\omega, u)$. (d) Significant values (in red) of the empirical wavelet coherence that are above the threshold $r_{\alpha}$. (e) Empirical WCS $\left|\hat{S}_{\mathbf{x y}}(\omega, u)\right|^{2}$. (f) Significant values (in red) of the empirical WCS that are above the threshold $\hat{\lambda}_{\alpha}$ - The value of $\alpha$ is $5 \%$ for both thresholds.

with $n=10$ is given in Figure 4. It can be seen that the test finds many significant values for the wavelet coherence which is clearly not consistent with the data from model (3.2). Consider now the results of our test on Examples 2. One can see from Figure 4 that all the values of the empirical WCS fall below the data-based threshold $\hat{\lambda}_{\alpha}$. Therefore, contrary to the test based on the empirical wavelet coherence, our test does not find significant values for the WCS which is consistent with model (3.2).

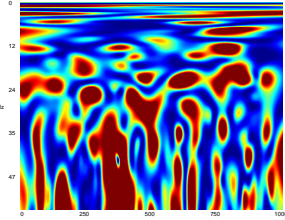

(a)

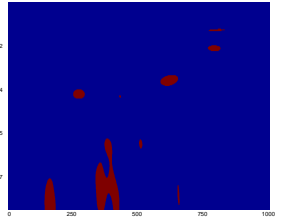

(b)

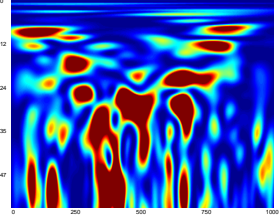

(c)

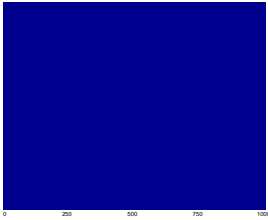

(d)

Figure 4: Example 2 - Automatic detection of time-frequency dependence using $n=10$ trials with $\mathrm{SNR}=-10 d B$. (a) Empirical wavelet coherence $\hat{R}_{\mathbf{x y}}^{2}(\omega, u)$. (b) Significant values (in red) of the empirical wavelet coherence that are above the threshold $r_{\alpha}$. (c) Empirical WCS $\left|\hat{S}_{\mathbf{X y}}(\omega, u)\right|^{2}$. (d) No significant values are found for the empirical WCS using the threshold $\hat{\lambda}_{\alpha}$ - The value of $\alpha$ is $5 \%$ for both thresholds.

Example 3 - Let us simulate again $n$ independent realizations (trials) $\left(\mathbf{x}_{m}\right)_{m=1, \ldots, n}$ and $\left(\mathbf{y}_{m}\right)_{m=1, \ldots, n}$ of the two times series $\mathbf{x}$ and $\mathbf{y}$ from model (3.1) with a SNR equal to $-5 d B$. Then, we apply data shuffling to the trials from the second time serie to artificially create independence across samples. 
More precisely, we propose to compare the detection of time-frequency dependence when computing the wavelet coherence and the WCS either from the raw data $\left(\mathbf{x}_{m}\right)_{m=1, \ldots, n}$ and $\left(\mathbf{y}_{m}\right)_{m=1, \ldots, n}$ or from the shuffled trials $\left(\mathbf{x}_{m}\right)_{m=1, \ldots, n}$ and $\left(\tilde{\mathbf{y}}_{m}\right)_{m=1, \ldots, n}$ where $\tilde{\mathbf{y}}_{m}=\mathbf{y}_{m+1}$ (see Figure 5 for an example with $n=30$ ). By data shuffling, the time series $\mathbf{x}_{m}$ and $\tilde{\mathbf{y}}_{m}$ are independent samples, and it is thus expected there is no coherence between such signals. It can be seen in Figure 5 (f) that the test on coherence detection finds many significant values for the wavelet coherence using the shuffled data which is clearly not satisfactory. To the contrary, Figure $5(\mathrm{~h})$ shows that our procedure does not find significant values for the WCS when using the shuffled data while still performing a consistent estimation of the WCS when using the raw data, see Figure 5(d). When the number of trials is small (see Figure 6(h) with $n=10$ ), the results of the data shuffling show remaining significant values of the WCS which are due to large value of the auto-spectrum of $\mathbf{x}$ and $\mathbf{y}$, but with much smaller areas than without data shuffling, see Figure 6(d).

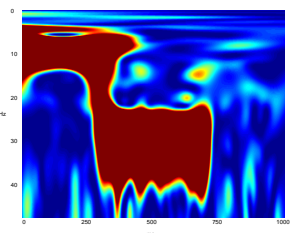

(a)

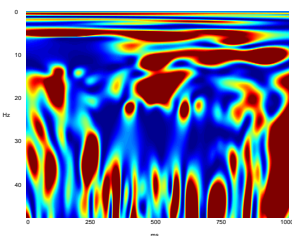

(e)

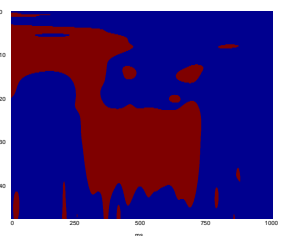

(b)

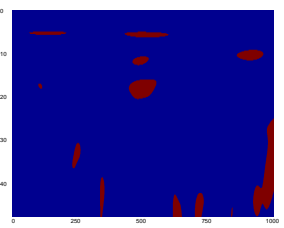

(f)

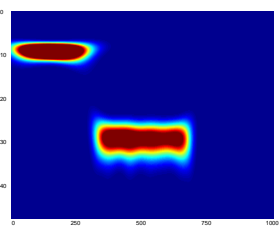

(c)

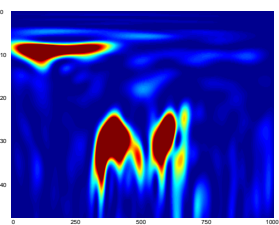

(g)

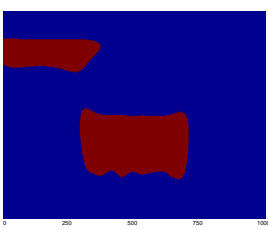

(d)

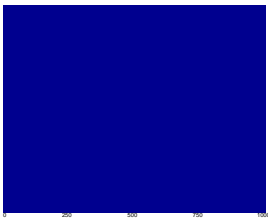

(h)

Figure 5: Example 3 - Automatic detection of time-frequency dependence using $n=30$ trials using either the raw data (first line) or the shuffled data (second line) (a,e) Empirical wavelet coherence $\hat{R}_{\mathbf{x y}}^{2}(\omega, u)$. (b,f) Significant values (in red) of the empirical wavelet coherence that are above the threshold $r_{\alpha}$. (c,g) Empirical WCS $\left|\hat{S}_{\mathbf{x y}}(\omega, u)\right|^{2}$. (d,h) Significant values (in red) of the empirical WCS that are above the threshold $\hat{\lambda}_{\alpha}$ - The value of $\alpha$ is $5 \%$ for both thresholds.

\subsubsection{Robustness to Gaussianity}

The derivation of the threshold $\hat{\lambda}_{\alpha}$ relies on the assumption that the time series are Gaussian processes. Therefore, one may wonder if the test is robust to such an hypothesis.

Example 4 - To study robustness to Gaussianity, we simulate $n$ independent realizations (trials) of the following times series of length $T=1000 \mathrm{~ms}$ with sampling rate $1 \mathrm{kHz}$

$$
\begin{aligned}
x\left(t_{k}\right) & =Z\left(\sin \left(2 \pi \omega_{1} t_{k}\right) \mathbb{1}_{\left[0, u_{1}[\right.}\left(t_{k}\right)+\sin \left(2 \pi \omega_{2} t_{k}\right) \mathbb{1}_{\left[u_{1}, u_{2}[\right.}\left(t_{k}\right)\right)+\sigma_{x} \epsilon_{1, k} \\
y\left(t_{k}\right) & =Z\left(a_{1} \sin \left(2 \pi \omega_{1} t_{k}\right) \mathbb{1}_{\left[0, u_{1}[\right.}\left[t_{k}\right)+a_{2} \sin \left(2 \pi \omega_{2} t_{k}\right) \mathbb{1}_{\left[u_{1}, u_{2}[\right.}\left(t_{k}\right)\right)+\sigma_{y} \epsilon_{2, k}, \\
\text { where } Z & \sim \operatorname{Laplace}(0,1) \text { and } \epsilon_{1, k} \sim_{\text {iid }} \operatorname{Laplace}(0,1) \epsilon_{2, k} \sim_{\text {iid }} \operatorname{Laplace}(0,1)
\end{aligned}
$$




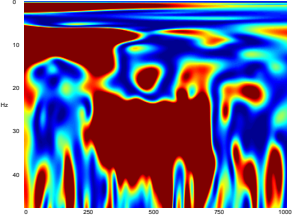

(a)

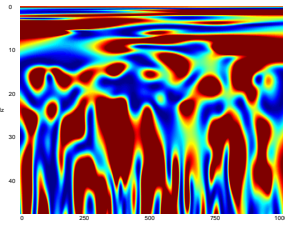

(e)

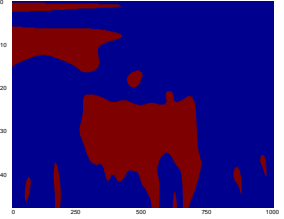

(b)

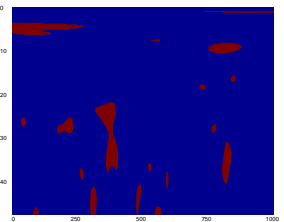

(f)

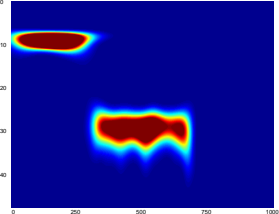

(c)

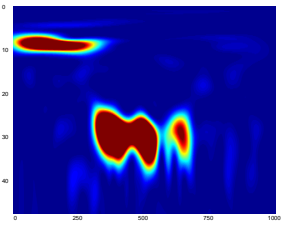

(g)

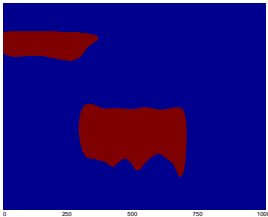

(d)

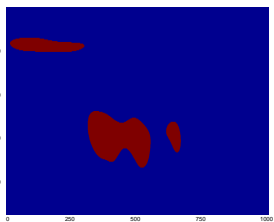

(h)

Figure 6: Example 3 - Automatic detection of time-frequency dependence using $n=10$ trials using either the raw data (first line) or the shuffled data (second line) (a,e) Empirical wavelet coherence $\hat{R}_{\mathbf{x y}}^{2}(\omega, u)$. (b,f) Significant values (in red) of the empirical wavelet coherence that are above the threshold $r_{\alpha}$. (c,g) Empirical WCS $\left|\hat{S}_{\mathbf{x y}}(\omega, u)\right|^{2}$. (d,h) Significant values (in red) of the empirical WCS that are above the threshold $\hat{\lambda}_{\alpha}$ - The value of $\alpha$ is $5 \%$ for both thresholds.

with $u_{1}, u_{2}, \omega_{1}, \omega_{2}, a_{1}, a_{2}, \sigma_{x}, \sigma_{y}$ chosen as in model (3.1), and where $\operatorname{Laplace}(0,1)$ denotes a random variable following a Laplace distribution with zero mean and variance one. The time series in model (3.3) thus follows a Laplace distribution, and are such that they have the same wavelet coherence and WCS than the Gaussian time series from model (3.1) which are displayed in Figure 1(d,e).

An example of realization from model (3.3) with a SNR equal to $-5 \mathrm{~dB}$ is displayed in Figure 7(a). It can be seen that time series following such a Laplace distribution are signals which contains isolated peaks. Hence, when compared to the Gaussian signals from model (3.1) in Figure 1(a), they are more appropriate to model spiky processes. The results of our testing procedure displayed in Figure Z(g) are very satisfactory. The test correctly estimates the areas in the time-frequency plane where the dependence between the time series is truly significant. Moreover, it does not detect any area where no correlation between the signals exists. Again, the results using the standard test to detect significant values of the wavelet coherence are not so satisfactory, see Figure $7(\mathrm{e})$.

\subsubsection{Evaluation of type I and type II errors: effects of $n$ and SNR}

To test the performances of this new procedure to detect significant values of time-frequency dependence between random signals, we propose to generate time series from model (3.1) using different values for the SNR and the number of trials, and to compare the results with those given by the standard test in $\mathrm{GC} 88, \mathrm{ZHJ}^{+} 06$ to detect significant values of wavelet coherence. Recall that the quality of a statistical test is expressed in terms of its type I and type II error rate. The type I error rate is the probability of a false positive, rejecting the null hypothesis at frequency-time point $(w, u)$ when it is true. The type II error rate is the probability of a false negative i.e. accepting the null hypothesis at frequency-time point $(w, u)$ when there is a truly significant level of time-frequency dependence at $(\omega, u)$ between the two-time series. The goal of this simulation study is to evaluate the type I and type II errors of the two tests at various points $(w, u)$ in the time-frequency plane. 


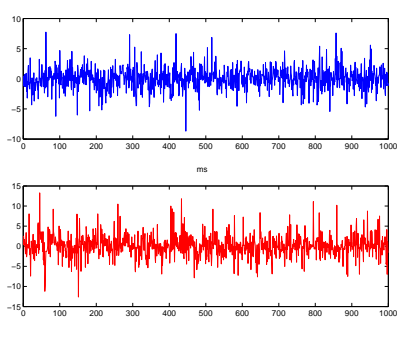

(a)

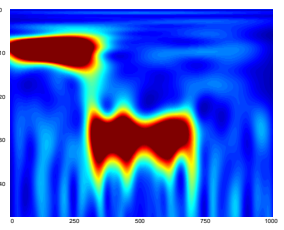

(b)

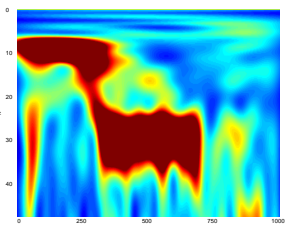

(c)

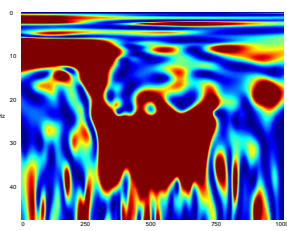

(d)

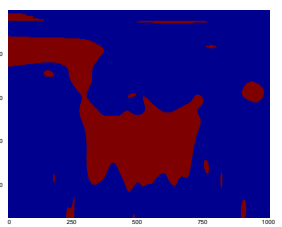

(e)

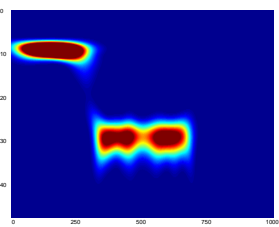

(f)

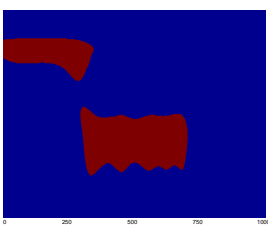

(g)

Figure 7: Example 4 - Robutness to Gaussianity using $n=10$ trials with $\mathrm{SNR}=-5 d B$. (a) An example of time series $\mathbf{x}_{1}$ and $\mathbf{y}_{1}$ generated from model (3.3). (b) Empirical wavelet auto-spectum $\hat{S}_{\mathbf{X}}(\omega, u)$. (c) Empirical wavelet auto-spectum $\hat{S}_{\mathbf{y}}(\omega, u)$. (d) Empirical wavelet coherence $\hat{R}_{\mathbf{x y}}^{2}(\omega, u)$. (e) Significant values (in red) of the empirical wavelet coherence that are above the threshold $r_{\alpha}$. (f) Empirical WCS $\left|\hat{S}_{\mathbf{x y}}(\omega, u)\right|^{2}$. (g) Significant values (in red) of the empirical WCS that are above the threshold $\hat{\lambda}_{\alpha}$ - The value of $\alpha$ is $5 \%$ for both thresholds.

The different values for the factors in the simulations are SNR $=-10,-20 d B$ and $n=10,100$ trials. For each combination of these two factors, we compare the two tests on $M=100$ repetitions from model (3.1). For $m=1, \ldots, M$, each repetition $m$ consists in simulating $n$ trials from model (3.1) for a given level of SNR. Then, based on these $n$ trials, one constructs two time-frequency testing maps $T_{m}(\omega, u)$ (one for each test) containing the result of each statistical test with $T_{m}(\omega, u)=0$ if the test accepts the null hypothesis $H_{0}$, and $T_{m}(\omega, u)=1$ if the test rejects $H_{0}$. For some repetition $m$, it is possible that the two (or only one) tests perform poorly or very good. It is therefore important to quantify the behavior of such tests on average, and not to draw conclusions from a single set of $n$ trials.

For this purpose, the performances of each test, over the $M=100$ repetitions, can be visualized from the following averaged time-frequency testing map:

$$
\bar{T}(\omega, u)=\frac{1}{M} \sum_{m=1}^{M} T_{m}(\omega, u) .
$$

Note that $\bar{T}(\omega, u)$ is a value between 0 and 1 that can be interpreted as the probability that the test rejects the null hypothesis $H_{0}$ at frequency-time point $(w, u)$. Values close to 1 indicate that (on average) the test rejects $H_{0}$ at frequency $\omega$ and time $u$, while values close to 0 indicate that (on average) the test accepts $H_{0}$. Therefore, comparing the values $\bar{T}(\omega, u)$ to the true WCS and the true wavelet coherence is way to evaluate the type I and type II errors of each test. Results are displayed in Figures 8 and 9, and the main comments that can be made are the following:

- as the SNR decreases the time-frequency maps given by the true wavelet coherence $R_{\mathbf{x y}}^{2}(\omega, u)$ and 
the true WCS $\left|S_{\mathbf{x y}}(\omega, u)\right|^{2}$ are more and more similar, compare Figures 1 (a)(b) with Figures $8,9(\mathrm{a})(\mathrm{d})$.

- the number of false positives using our test is extremely low. This means that a value of the empirical WCS that is above the threshold $\hat{\lambda}_{\alpha}$ can be considered as being a truly significant level of time-frequency dependence with a high confidence. To the contrary, the test on empirical wavelet coherence using the threshold $r_{\alpha}$ yields many false positive.

- when the signal-to-noise ratio is high $(\mathrm{SNR}=-10 \mathrm{db})$, our test performs very well with few trials $(n=10)$. For a higher SNR, the performances of our test are still very satisfactory when using more trials.

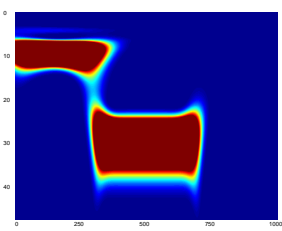

(a) True WC

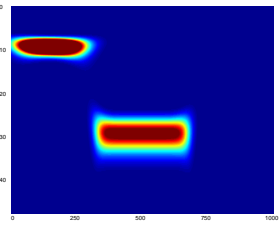

(d) True WCS

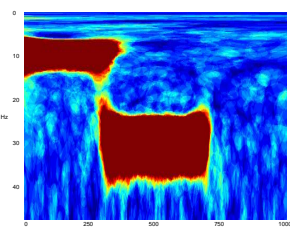

(b) $n=10$

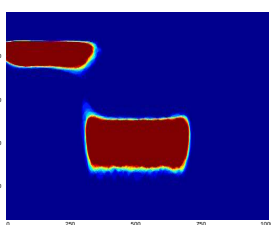

(e) $n=10$

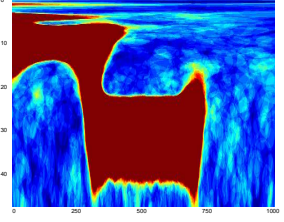

(c) $n=100$

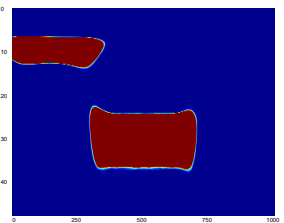

(f) $n=100$

Figure 8: Simulations with SNR $=-10 d B$. (a) True wavelet coherence $R_{\mathbf{x y}}^{2}(\omega, u)$. (d) True WCS $\left|S_{\mathbf{X y}}(\omega, u)\right|^{2}$. First row: averaged testing map for the detection of significant values of the wavelet coherence with (b) $n=10$ and (c) $n=100$. Second row: averaged testing map for the detection of significant values of the WCS with (e) $n=10$ and (f) $n=100$ (red indicates values close to 1 and blue indicates values close to 0$)$.

\subsection{Application to the analysis of corticomuscular interactions}

To assess the usefulness of the proposed approach with an experimental example, we compare the results of the two tests when applied to neurophysiological signals. Data were collected from a single healthy adult male volunteer, as part of a study on the effects of force level on corticomuscular interactions during submaximal voluntary isometric contractions. The participant was secured in a seated position with the right knee $60^{\circ}$ flexed on a calibrated dynamometer (System 4 Pro, Biodex Medical Systems, Shirley, NY, USA) used to record the net joint torque around the knee at 1000 $\mathrm{Hz}$. He was asked to perform blocks of isometric contractions of the right knee extensors at either $10 \%$ or $20 \%$ of previously determined maximal voluntary contraction (MVC), in a randomized order. Each contraction level was performed 10 times per block for 6 seconds, and was followed by a 6 -second rest interval. Each block was followed by 3-minutes of rest, and overall, 10 blocks 


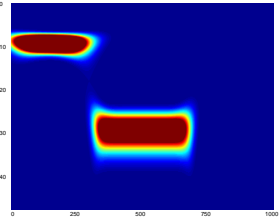

(a) True WC

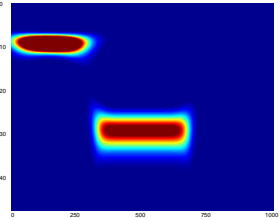

(d) True WCS

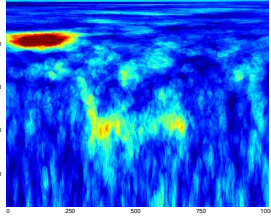

(b) $n=10$

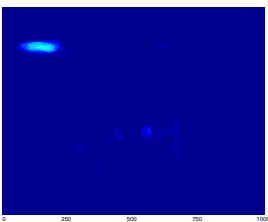

(e) $n=10$

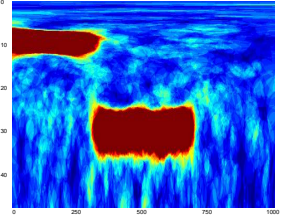

(c) $n=100$

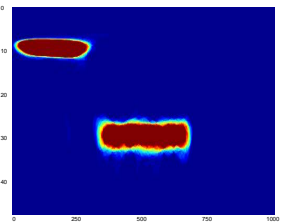

(f) $n=100$

Figure 9: Simulations with $\mathrm{SNR}=-20 d B$. (a) True wavelet coherence (WC) $R_{\mathbf{x y}}^{2}(\omega, u)$. (b) True WCS $\left|S_{\mathbf{x y}}(\omega, u)\right|^{2}$. First row: averaged testing map for the detection of significant values of the wavelet coherence with (b) $n=10$ and (c) $n=100$. Second row: averaged testing map for the detection of significant values of the WCS with (e) $n=10$ and (f) $n=100$ (red indicates values close to 1 and blue indicates values close to 0 ).

were performed leading to a total of 100 contractions per MVC level. The required MVC level was controlled through a visual torque feedback displayed on a screen placed one meter in front of the participant. The participant had to exert the required torque level as soon as torque feedback was provided, and to maintain it as accurately as possible through the duration of the contraction until disappearance of the torque feedback. During the contractions, he was required to keep its upper body and left lower limb muscles relaxed and its arms rested on each tight.

Electroencephalographic signal (EEG) was recorded reference-free at $1024 \mathrm{~Hz}$ using a 64-channel ActiveTwo system (BioSemi, Amsterdam, Netherlands; electrode impedances below $5 \mathrm{k}$ ), with electrodes arranged according to the International 10-20 system. The continuous EEG signal was highpass filtered at $0.5 \mathrm{~Hz}$ (zero-lag, 4th order Butterworth filter) and referenced to the $\mathrm{FCz}$ electrode. The $\mathrm{Cz}$ electrode was selected for further analysis as the electrode optimally located to record cerebral activity directly linked to right lower limb muscles contraction [MN08, PLJN06. Following suitable skin preparation HFDKR00, surface electromyographic signal (EMG) was recorded from Vastus Medialis (VM) at $1000 \mathrm{~Hz}$ using a Bagnoli-8 system (DE-2.1, Delsys, Inc., Boston, MA, USA) with the reference electrode on the left radial styloid. The continuous EMG was resampled to 1024 $\mathrm{Hz}$ by third-order spline interpolation and high-pass filtered at $3 \mathrm{~Hz}$ (zero-lag, 4th order Butterworth filter).

Continuous data were then epoched from $-1000 \mathrm{~ms}$ to $+7000 \mathrm{~ms}$ after the onset of the torque feedback, i.e., $\mathrm{T}=8192$ for each contraction. After rejection of the trials contaminated by EEG and/or EMG artefacts, the number of remaining contraction was $n=70$ for each MVC level. Thus, the value of the ratio $T / n$ is in agreement with the conditions discussed in the Appendix in the sense that $T$ is much larger than $n$. 


\section{Experimental results}

Figure 10 (third to fifth column) present the values of the WCS and the wavelet coherence between EEG and EMG, and their corresponding significant values, for a large number of trials $(n=70)$. First, the results show that WCS and wavelet coherence contain different information. Large values of wavelet coherence are widely spread in the time-frequency plane whatever the MVC level, without clear difference in the correlation between EEG and EMG during the rest and contraction periods. To the contrary, large values of WCS are observed for non-null MVC levels in frequency bands centered at $10 \mathrm{~Hz}$ and $20 \mathrm{~Hz}$, specifically on the time interval of muscular contraction (knee extension). Secondly, the statistical test using the threshold $r_{\alpha}$ finds isolated significant values of the wavelet coherence, dispersed both in time and frequency, whatever the MVC level. The test detects significant areas in the time-frequency plane during rest periods and does not display a clear correlation between EEG and EMG during maintained knee extension. Opposite to these results, the proposed test using the threshold $\hat{\lambda}_{\alpha}$ reveals absence of correlation between EEG and EMG during rest periods and for $0 \%$ MVC, and finds bands of significant correlation centered at $10 \mathrm{~Hz}$ and $20 \mathrm{~Hz}$ during knee extension for $10 \%$ and $20 \%$ MVC. In addition to these highlighted features, the statistical test based on the thresholding of the WCS indicates clearer differences in the correlation between EEG and EMG with increased MVC level.

Another benefit of our approach is that it gives good results with very few trials. To illustrate this fact, we display the results obtained when using only $n=10$ trials (randomly chosen from the 70 trials) in Figure 11. Using a smaller number of trials $(n=10$, see Figure 11), similar differences are observed between the WCS and the wavelet coherence, and a similar trend is found between the two tests. Apart from this general result, the significant values of the wavelet coherence (threshold $r_{\alpha}$ ) indicate increased dispersion of the correlation between EEG and EMG in the time-frequency plane with $n=10$ than with $n=70$. When thresholding the WCS with $\hat{\lambda}_{\alpha}$, it can be seen from Figure 11(n) and 11)(r) that the decrease in the number of trials results i) in broader bands centered at $10 \mathrm{~Hz}$ and $20 \mathrm{~Hz}$ of significant correlation between EEG and EMG, and ii) in the detection of significant areas centered at $7 \mathrm{~Hz}$ during periods of rest, see Figure 11(j).

\section{Data shuffling}

For MVC $=10 \%$ and MVC $=20 \%$, Figure 10(c,e) show large variations of the EEG WAS power specifically in the frequency bands of interest centered at $10 \mathrm{~Hz}$ and $20 \mathrm{~Hz}$ (i.e., alpha and beta bands, respectively), when compared to the case $\mathrm{MVC}=0 \%$, Figure 10(a). This marked variation in the autospectra causes variation in the WCS, and one may wonder if our test automatically adapts to the change in magnitude of the WCS while still allowing a consistent detection of the significant values of the WCS. To illustrate this point, we used data shuffling as follows. We have compared the results of our test when computing either a WCS using the EEG trials at MVC $=0 \%$ and the EMG trials at MVC $=0 \%$ or a WCS using the EEG trials at MVC $=20 \%$ and the EMG trials at MVC $=0 \%$. In both cases our test detects very few significant values in the WCS (see Figure 12(d) and compare with Figure 10(j)). 


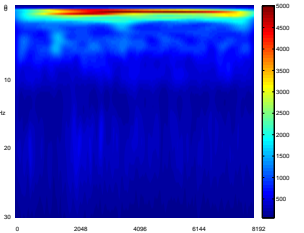

(a) WAS EEG / 0\%

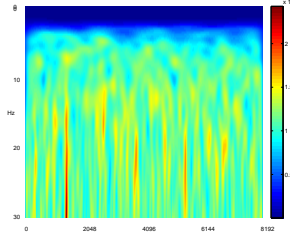

(b) WAS EMG / 0\%

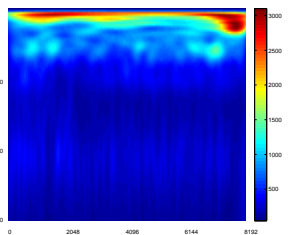

(e) WAS EEG / $20 \%$

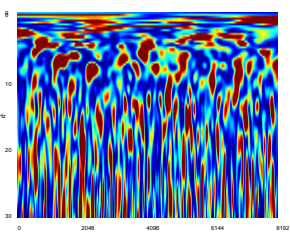

(g) $\mathrm{WC} / 0 \%$

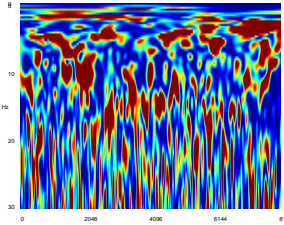

(k) WC / $10 \%$

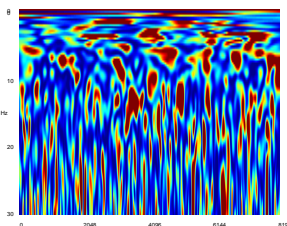

(o) $\mathrm{WC} / 20 \%$

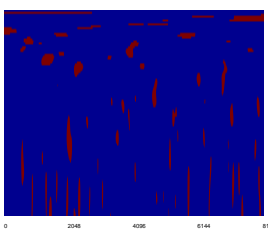

(h) Test WC / 0\%

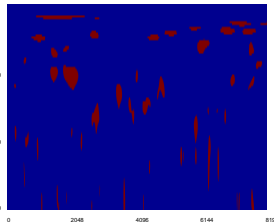

(l) Test WC / 10\%

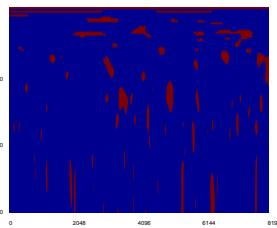

(p) Test WC / 20\%

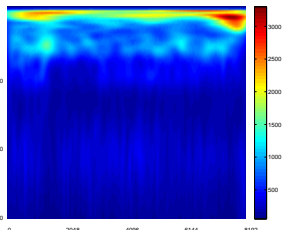

(c) WAS EEG / 10\%

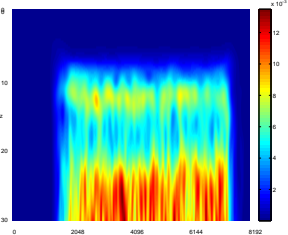

(f) WAS EMG / $20 \%$

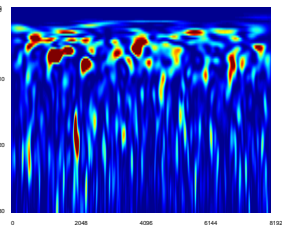

(i) WCS / $0 \%$

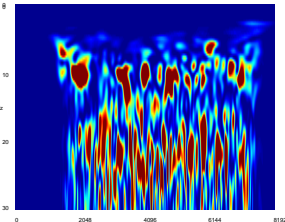

(m) WCS / $10 \%$

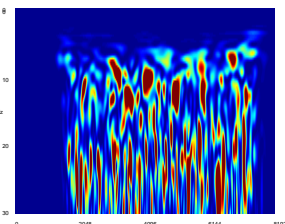

(q) WCS / $20 \%$

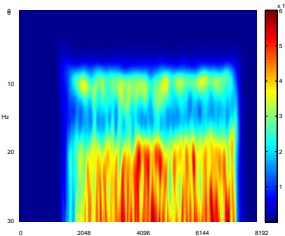

(d) WAS EMG / 10\%

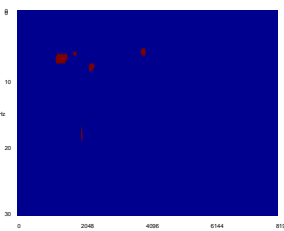

(j) Test WCS / 0\%

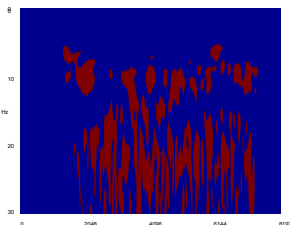

(n) Test WCS / 10\%

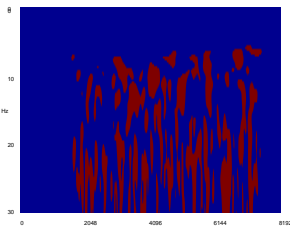

(r) Test WCS / 20\%

Figure 10: Analysis of wavelet coherence and WCS on EEG and EMG during isometric contractions of the knee extensors using $n=70$ trials. First row : empirical wavelet auto-spectra (WAS) of EEG and $\mathrm{EMG}$ for $\mathrm{MVC}=0 \%$, (no efforts) and MVC $=10 \%$. Second row : WAS of EEG and EMG for $\mathrm{MVC}=20 \%$. Third row : data MVC $=0 \%$, (no efforts), fourth row : data for MVC $=10$ $\%$, fifth row data for $\mathrm{MVC}=20 \%$. First column: empirical wavelet coherence $\hat{R}_{\mathbf{x y}}^{2}(\omega, u)$. Second column: significant values (in red) of the empirical wavelet coherence that are above the threshold $r_{\alpha}$. Third column: empirical WCS $\left|\hat{S}_{\mathbf{x y}}(\omega, u)\right|^{2}$. Fourth column: significant values (in red) of the empirical WCS that are above the threshold $\hat{\lambda}_{\alpha}$. 


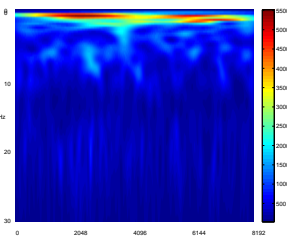

(a) WAS EEG / 0\%

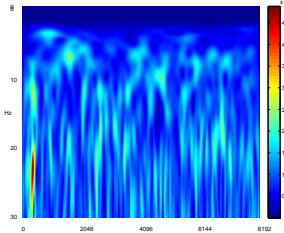

(b) WAS EMG / 0\%

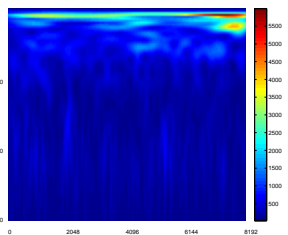

(e) WAS EEG / $20 \%$

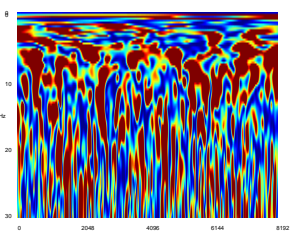

(g) $\mathrm{WC} / 0 \%$

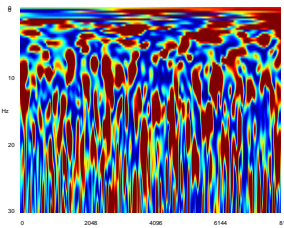

(k) WC / $10 \%$

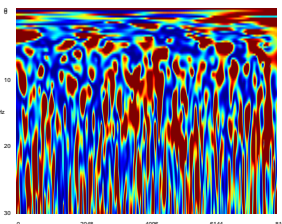

(o) $\mathrm{WC} / 20 \%$

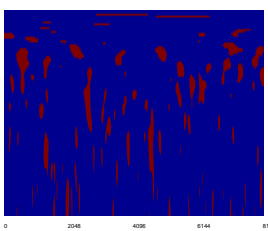

(h) Test WC / 0\%

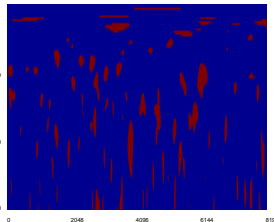

(l) Test WC / $10 \%$

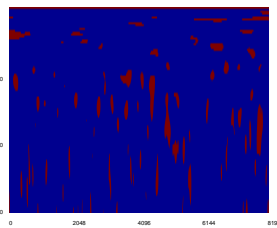

(p) Test WC / 20\%

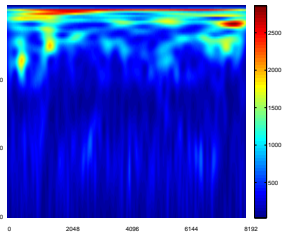

(c) WAS EEG / $10 \%$

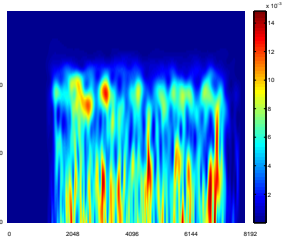

(f) WAS EMG / $20 \%$

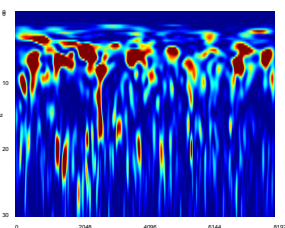

(i) WCS / $0 \%$

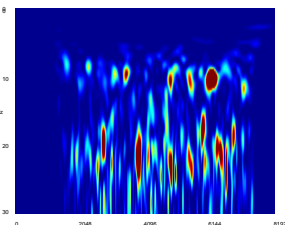

(m) WCS / $10 \%$

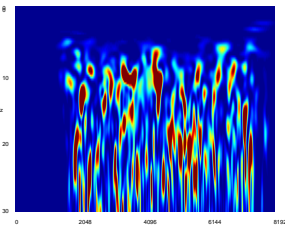

(q) WCS / $20 \%$

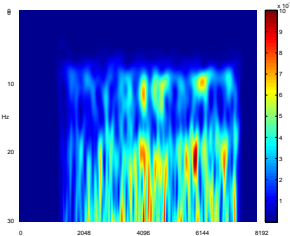

(d) WAS EMG / 10\%

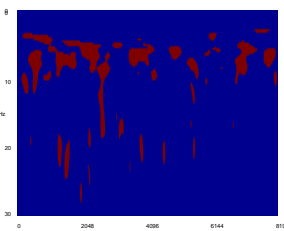

(j) Test WCS / 0\%

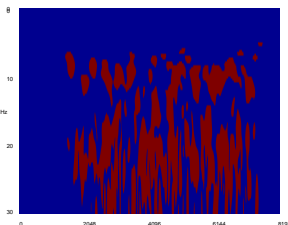

(n) Test WCS / 10\%

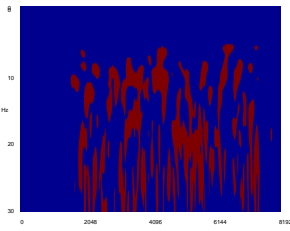

(r) Test WCS / 20\%

Figure 11: Analysis of wavelet coherence and WCS on EEG and EMG during isometric contractions of the knee extensors using only $n=10$ trials. First row : empirical wavelet auto-spectra (WAS) of EEG and EMG for MVC $=0 \%$, (no efforts) and MVC $=10 \%$. Second row : WAS of EEG and EMG for MVC $=20 \%$. Third row : data MVC $=0 \%$, (no efforts), fourth row : data for MVC = $10 \%$, fifth row data for $\mathrm{MVC}=20 \%$. First column: empirical wavelet coherence (WC) $\hat{R}_{\mathbf{x y}}^{2}(\omega, u)$. Second column: significant values (in red) of the empirical wavelet coherence that are above the threshold $r_{\alpha}$. Third column: empirical WCS $\left|\hat{S}_{\mathbf{x y}}(\omega, u)\right|^{2}$. Fourth column: significant values (in red) of the empirical WCS that are above the threshold $\hat{\lambda}_{\alpha}$. 


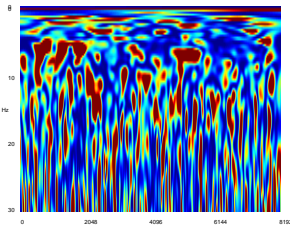

(a) WC Shuf

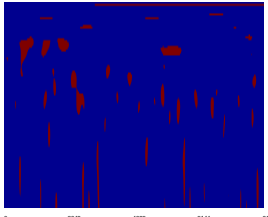

(b) Test WC Shuf

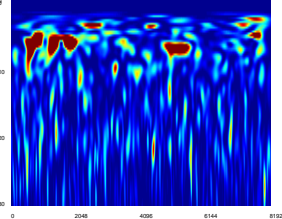

(c) WCS Shuf

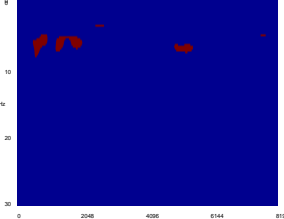

(d) Test WCS Shuf

Figure 12: Analysis of wavelet coherence and WCS on EEG and EMG during isometric contractions of the knee extensors using $n=70$ trials and data shuffling. First row : data shuffling (EEG $\mathrm{MVC}=20 \% / \mathrm{EMG}-\mathrm{MVC}=0 \%)$. First column: empirical wavelet coherence $\hat{R}_{\mathbf{x y}}^{2}(\omega, u)$. Second column: significant values (in red) of the empirical wavelet coherence that are above the threshold $r_{\alpha}$. Third column: empirical WCS $\left|\hat{S}_{\mathbf{x y}}(\omega, u)\right|^{2}$. Fourth column: significant values (in red) of the empirical WCS that are above the threshold $\hat{\lambda}_{\alpha}$.

\section{Discussion}

\subsection{Theoretical comparison of the two statistical procedures}

Our method has been analyzed from the point of view of statistical hypothesis testing. With small probability, the procedure rejects the null hypothesis that the repeated trials come from two independent Gaussian time series. The method is thus valid for the analysis of any Gaussian processes with zero mean, in particular for those that are not stationary. Its use is therefore more general than the standard test for significant wavelet coherence detection which is mainly valid for the null hypothesis that the two time series are independent white noise.

\subsection{Comparison using simulated data}

First, if the level of noise in the measurements is high, then the "true" wavelet coherence and the "true" WCS tend to carry the same kind of information on time-frequency dependence between two time series. However, the testing procedures using either the empirical wavelet coherence or the empirical WCS clearly yield to very different conclusions on the nature of the correlations between two times series.

The numerical experiments show that the use of the standard test using wavelet coherence yields erroneous coherence detection (type I error in statistical hypothesis testing), and can miss truly significant values (type II error in statistical hypothesis testing). In particular, this test detects areas the time-frequency plane where no correlation between the signals exists. Indeed, for all values of SNR and numbers of trials, the test using the wavelet coherence yields many false positive, which clearly questions the interpretability of this test and its level of confidence. To the contrary, our procedure correctly estimates the areas in the time-frequency plane where the dependence between the time series is truly significant. Moreover, our test does not detect any area where no correlation between the signals exists, meaning that our test is more conservative.

These results using data shuffling support the argument that our test is adapted to the case where the auto-spectrum of each time serie can be very large. Hence, a value of the WCS above the threshold $\hat{\lambda}_{\alpha}$ can generally be considered as being due to time-frequency dependence between the signals.

With very few trials, the results obtained using the test on wavelet coherence detection are 
extremely unsatisfactory. To the contrary, our procedure to detect significant values of the crossspectrum performs very well with few repeated observations. Indeed, the results obtained with only $n=2$ trials clearly show that our test correctly estimates the areas in the time-frequency plane where the dependence between the time series is truly significant, and does not detect any area where no correlation between the signals exists.

The numerical examples also demonstrate some robustness of the test to the assumption that the time series should be Gaussian.

To the best of our knowledge, this testing procedure to detect significant values of the crossspectrum is new, and we believe that it represents a powerful alternative to some limitations of wavelet coherence analysis.

\subsection{Discussion on the analysis of corticomuscular interactions}

Our results on the analysis of the correlation between EEG and EMG, i.e. the corticomuscular interactions, emphasize those obtained from simulated data and illustrate the potential of the proposed test to effectively detect time-frequency dependence between non-stationary signals. Thresholding the WCS with the data-based threshod $\hat{\lambda}_{\alpha}$ overcomes the effects of both time and frequency scaling observed when thresholding the wavelet coherence with $r_{\alpha}$. In agreement with previous findings on corticomuscular synchronization (for review, see [MH99a, SH03]), the proposed statistical test improves the detection of the significant areas of correlation at $10 \mathrm{~Hz}$ and $20 \mathrm{~Hz}$ between EEG and EMG in the time-frequency plane. Significant corticomuscular interactions were found around 20 $\mathrm{Hz}$ in agreement with the literature (see $\mathrm{CHF}^{+95}, \mathrm{KBS}^{+} 00, \mathrm{SPK}^{+} 97$ ) but also around $10 \mathrm{~Hz}$, a finding that is less common but nonetheless reported in several studies in healthy subjects (see [FAKF00, MBS01]) and in Parkinson's patients (see [RGM ${ }^{+}$09]).

Using our method, corticomuscular interaction was not significant during the rest periods and during $0 \%$ MVC trials. This feature is clearly an advantage of the proposed test for application to the analysis of corticomuscular interactions, because it does not detect significant area where no correlation exists between the non-stationary signals. For non-null MVC levels, the statistical test on the WCS with $\hat{\lambda}_{\alpha}$ does not find significant peaks of coherence dispersed in the time-frequency plane. To the contrary, when the number of trials is high $(n=70)$, the proposed test detects accurate bands of correlation centered at $10 \mathrm{~Hz}$ and $20 \mathrm{~Hz}$ during the time interval of muscular contraction. With regards to the results from simulated data, one can suggest that the proposed test estimates more correctly the areas in the time-frequency plane where the dependence between EEG and EMG is truly significant, and that it offers the major advantage to reduce the detection of false positive when compared with the test on the wavelet coherence with $r_{\alpha}$.

Another benefit of our approach for application to the analysis of corticomuscular interactions is that the improvement of the detection of corticomuscular interactions is maintained with a small number of trials. Despite an observed increased dispersion in frequency with few trials $(n=10)$, the results obtained using the proposed test conserve similar general features than those observed with a large number of trials, which illustrates the practical advantage of our approach when the clinical conditions limit the possible number of experimental trials.

Finally, using data shuffling, we have shown that our method automatically includes an estimation of the variance of the two time series in the computation of the threshold used to detect significant value of the WCS. Indeed, although in the case EEG - MVC $=0 \% / \mathrm{EMG}-\mathrm{MVC}=0 \%$ the magnitude of the WCS increases, our test does not detect more significant time-frequency dependence which is consistent with the fact that connectivity does not change when compared to the case EEG - MVC 
$=20 \% / \mathrm{EMG}-\mathrm{MVC}=0 \%$.

\subsection{Advantages of WCS in contrast with wavelet coherence}

Although the cross-spectrum is a non-normalized measure of dependence, our testing procedure can be used to detect significant values of the empirical WCS since it automatically estimates the amplitude of the auto-spectra of the time series. Coherence is often considered as being more interpretable since it is a normalized measure. However, results in this paper clearly show that a significant value of wavelet coherence (above the detecting threshold $r_{\alpha}$ ) does not necessarily correspond to a significance level of time-frequency dependence. Hence, the use of the standard test on wavelet coherence may lead to erroneous conclusions. To the contrary, in many situations, the use of the WCS combined with our testing procedure is a much more reliable way to detect areas in the time-frequency plane where the dependence between two time series is truly significant. Although we have demonstrated some robustness to Gaussianity, a limitation of our approach is that the derivation of the threshold $\lambda_{\alpha}$ relies on the assumption that the time series are Gaussian.

\section{Conclusion}

We have proposed a new statistical test to detect significant values of the wavelet cross-spectrum between two time series using repeated trials. These values correspond to a truly significant level of time-frequency dependence between the two time series. The test is a fully data-driven procedure based a simple thresholding of the wavelet cross-spectrum. Throughout the paper, this method has been compared with the standard test described in the literature to detect significant values of wavelet coherence between two time series. Comparisons have been made using both theoretical arguments and numerical experiments.

In usual experiments in neuroscience, one often wants to know if a WCS is statistically different from another WCS. This corresponds to the null hypothesis that the difference between two WCS computed from two data sets consisting of pair of time series is zero. A natural procedure to test such an hypothesis would be to compute the difference between two empirical WCS, and then to use an appropriate threshold to detect significant values of the resulting time-frequency map. We believe that the theoretical arguments developed in this paper could be used to derive such a threshold, which represents an interesting topic for future work.

Acknowledgements - This work was supported by the research project grant AO3 NeuroBiomeCo from the University Paul Sabatier. J. Bigot would like to thank the Center for Mathematical Modeling and the CNRS for financial support and excellent hospitality while visiting Santiago where part of this work was carried out. Numerical experiments have been implemented using the MATLAB programming environment and the MATLAB package provided by Aslak Grinsted for performing cross-wavelet and wavelet coherence analysis which can be downloaded at:

http://www.pol.ac.uk/home/research/waveletcoherence/ 


\section{Appendix}

\subsection{Derivation of the threshold $\hat{\lambda}_{\alpha}$}

The following proposition shows that it is possible to derive under $H_{0}\left(\Sigma_{x}, \Sigma_{y}\right)$ a probabilistic upper bound for the empirical WCS (the proof is given in Section 5.2). The key quantities to control such an upper bound are the maximal eigenvalues $\rho_{x}^{2}$ and $\rho_{y}^{2}$ of the covariance matrices $\Sigma_{x}$ and $\Sigma_{y}$ defined as $\rho_{x}^{2}=\max _{v \in \mathbb{R}^{T}} \frac{v^{\prime} \Sigma_{x} v}{v^{\prime} v}$ and $\rho_{y}^{2}=\max _{v \in \mathbb{R}^{T}} \frac{v^{\prime} \Sigma_{y} v}{v^{\prime} v}$. Note that in the case where $\Sigma_{x}=\sigma_{x}^{2} I_{T}$ and $\Sigma_{y}=\sigma_{y}^{2} I_{T}$ then $\rho_{x}^{2}=\sigma_{x}^{2}$ and $\rho_{y}^{2}=\sigma_{y}^{2}$.

Proposition 5.1 Suppose that the hypothesis $H_{0}\left(\Sigma_{x}, \Sigma_{y}\right)$ is true. Let $0<\alpha<1$. Assume that $\psi$ is the Morlet wavelet defined in (2.1). For any frequency $\omega$ and time $u$, define the threshold

$$
\lambda_{\alpha}=\frac{\rho_{x} \rho_{y}}{n}\left\|\psi_{\omega, u}\right\|^{2}(-\log (\alpha / 2)+\sqrt{-2 n \log (\alpha / 2)}),
$$

where $\left\|\psi_{\omega, u}\right\|^{2}=\sum_{k=1}^{T}\left|\psi_{\omega, u}\left(t_{k}\right)\right|^{2}$ and $\psi_{\omega, u}\left(t_{k}\right)=\sqrt{\frac{\omega}{\omega_{0}}} \psi\left(\frac{\omega}{\omega_{0}}\left(t_{k}-u\right)\right)$. Then, for any $n \geq 1$

$$
\mathbb{P}\left(\left|\hat{S}_{\mathbf{x y}}(\omega, u)\right|>\lambda_{\alpha}\right) \leq \alpha
$$

where for a random variable $Z$ and a real $t>0$, the notation $\mathbb{P}(|Z|>t)$ denotes the probability of the event that the modulus of $Z$ is greater than $t$.

In all the numerical experiments of the paper, the energy (or $L^{2}$ norm) of the wavelet $\psi$ is normalized to be one at all scales (meaning that $\left\|\psi_{\omega, u}\right\|^{2}=1$ ). Under such an assumption, the threshold $\lambda_{\alpha}$ does not depend on the frequency $\omega$ and time $u$, and one can take the simplified threshold

$$
\lambda_{\alpha}=\frac{\rho_{x} \rho_{y}}{n}(-\log (\alpha / 2)+\sqrt{-2 n \log (\alpha / 2)}) .
$$

Note also that Proposition 5.1 can be applied with any mother wavelet $\psi$ that is a real-valued function. However, this procedure is obviously not directly applicable to real data, as the covariance matrices $\Sigma_{x}$ and $\Sigma_{y}$ and thus the eigenvalues $\rho_{x}^{2}$ and $\rho_{y}^{2}$ are typically unknown in practice. Nevertheless, data-based values for these parameters can be given. Indeed, if one observes $n$ repeated trials $\left(\mathbf{x}_{m}\right)_{m=1, \ldots, n}$ and $\left(\mathbf{y}_{m}\right)_{m=1, \ldots, n}$ (viewed as $n$ independent realizations of the stochastic processes $\mathbf{x}$ and $\mathbf{y}$ respectively) then one can define unbiased estimators of $\Sigma_{x}$ and $\Sigma_{y}$ by taking the following empirical covariance matrices

$$
\widehat{\Sigma}_{x}=\frac{1}{n} \sum_{m=1}^{n} \mathbf{x}_{m} \mathbf{x}_{m}^{\prime} \text { and } \widehat{\Sigma}_{y}=\frac{1}{n} \sum_{m=1}^{n} \mathbf{y}_{m} \mathbf{y}_{m}^{\prime} .
$$

It is then tempting to estimate $\rho_{x}^{2}$ and $\rho_{y}^{2}$ by the maximal eigenvalues $\hat{\rho}_{x}^{2}$ and $\hat{\rho}_{y}^{2}$ of the empirical covariance matrices $\widehat{\Sigma}_{x}$ and $\widehat{\Sigma}_{y}$ defined as

$$
\hat{\rho}_{x}^{2}=\max _{v \in \mathbb{R}^{T}} \frac{v^{\prime} \widehat{\Sigma}_{x} v}{v^{\prime} v} \text { and } \hat{\rho}_{y}^{2}=\max _{v \in \mathbb{R}^{T}} \frac{v^{\prime} \widehat{\Sigma}_{y} v}{v^{\prime} v} .
$$

Note that the quantities $\hat{\rho}_{x}^{2}$ and $\hat{\rho}_{y}^{2}$ are not difficult to compute numerically using standard software such as the MATLAB programming environment. However, results from random matrix theory (see 
[EK07] and references therein) show that $\hat{\rho}_{x}^{2}$ and $\hat{\rho}_{y}^{2}$ are not consistent estimators of $\rho_{x}^{2}$ and $\rho_{y}^{2}$. Indeed, in the case where $\Sigma_{x}=\sigma_{x}^{2} I_{T}$ and $\Sigma_{y}=\sigma_{y}^{2} I_{T}$ then

$$
\lim _{n \rightarrow+\infty, T \rightarrow+\infty} \hat{\rho}_{x}=\sigma_{x}(1+\sqrt{\gamma}) \text { and } \lim _{n \rightarrow+\infty, T \rightarrow+\infty} \hat{\rho}_{y}=\sigma_{y}(1+\sqrt{\gamma})
$$

where $\gamma=\lim _{n \rightarrow+\infty, T \rightarrow+\infty} \frac{T}{n}$. Therefore if $\gamma>0$ then $\hat{\rho}_{x}$ does not converge to $\sigma_{x}$. The coefficient $\gamma$ reflects the ratio between the length $T$ of the time series and the number of trials $n$. Typically, $T$ is much larger than $n$ and in practice, the ratio $\frac{T}{n}$ can be larger than 10 or 100, meaning that the ratio $\frac{\hat{\rho}_{x}}{\sigma_{x}} \approx(1+\sqrt{\gamma})$ is not close to one. This phenomena is a well known problem for the statistical estimation of large covariance matrices (see e.g. BL08 and references therein) in the high-dimensional data setting when the size of the data (here the number of time points $T$ ) is much larger than the number of repeated observations $n$.

In the more general case where $\Sigma_{x} \neq \sigma_{x}^{2} I_{T}$ or $\Sigma_{y} \neq \sigma_{y}^{2} I_{T}$, using Theorem II.13 in [DS01] it can be shown that for any value of $0<\beta \leq 1$ and any fixed $n \geq 1$ and $T \geq 1$ then

$$
\mathbb{P}\left(\hat{\rho}_{x} \geq \rho_{x}\left(1+\sqrt{\frac{T}{n}}+\sqrt{\frac{-2 \log (\beta)}{n}}\right)\right) \leq \beta .
$$

These results therefore suggest to estimate $\rho_{x}$ and $\rho_{y}$ by $\hat{\rho}_{x} /\left(1+\sqrt{\frac{T}{n}}\right)$ and $\hat{\rho}_{y} /\left(1+\sqrt{\frac{T}{n}}\right)$ which leads to the use of the following data-based threshold $\hat{\lambda}_{\alpha}$ (in the case where the energy of the wavelet $\psi$ is normalized to be one)

$$
\hat{\lambda}_{\alpha}=\frac{\hat{\rho}_{x} \hat{\rho}_{y}}{\left(1+\sqrt{\frac{T}{n}}\right)^{2}}\left(-\frac{\log (\alpha / 2)}{n}+\sqrt{-\frac{2 \log (\alpha / 2)}{n}}\right) .
$$

\subsection{Proof of Proposition 5.1}

Let $Z=\hat{S}_{\mathbf{x y}}(\omega, u)$ and remark that under $H_{0}$ the random variable $Z$ can be written as

$$
Z=\frac{1}{n} \sum_{m=1}^{n} X_{m, 1}^{\prime} \Sigma_{x}^{1 / 2} a \overline{a^{\prime}} \Sigma_{y}^{1 / 2} X_{m, 2}
$$

where $X_{m, 1}$ and $X_{m, 2}$ are independent centered Gaussian vector in $\mathbb{R}^{T}$ with covariance matrix the identity, and $a$ is the deterministic vector in $\mathbb{C}^{T}$ with entries

$$
a=\left[\psi_{\omega, u}\left(t_{k}\right)\right]_{k=1}^{T} \text { where } \psi_{\omega, u}\left(t_{k}\right)=\sqrt{\frac{\omega}{\omega_{0}}} \psi\left(\frac{\omega}{\omega_{0}}\left(t_{k}-u\right)\right) .
$$

Then, define the following vector $X \in \mathbb{R}^{n 2 T}$ by concatenation of the vectors $X_{m, 1}$ and $X_{m, 2}$ in the following way:

$$
X=\left(\begin{array}{c}
X_{m, 1} \\
X_{m, 2}
\end{array}\right)_{m=1, \ldots, n} \in \mathbb{R}^{n 2 T}
$$

Note that $X$ is a centered Gaussian vector with covariance matrix the identity. Then, define the $2 T \times 2 T$ matrix with complex entries

$$
A_{x y}=\frac{1}{2 n}\left(\begin{array}{cc}
\Sigma_{x}^{1 / 2} & 0 \\
0 & \Sigma_{x}^{1 / 2}
\end{array}\right)\left(\begin{array}{cc}
0 & a \overline{a^{\prime}} \\
\bar{a} a^{\prime} & 0
\end{array}\right)\left(\begin{array}{cc}
\Sigma_{y}^{1 / 2} & 0 \\
0 & \Sigma_{y}^{1 / 2}
\end{array}\right)
$$


and introduce the following $n 2 T \times n 2 T$ block-diagonal matrix

$$
A=\left(\begin{array}{cccc}
A_{x y} & 0 & \ldots & 0 \\
0 & A_{x y} & 0 & 0 \\
\vdots & 0 & \ldots & 0 \\
0 & 0 & 0 & A_{x y}
\end{array}\right)
$$

Given the definition (2.1) of the Morlet wavelet $\psi$, one can check that the matrix $a \overline{a^{\prime}}$ is Hermitian which implies that the matrices $A_{x y}$ and $A$ are Hermitian. Then, one can remark that the random variable $Z$ can be written in the form of a $\chi^{2}$ variable as

$$
Z=X^{\prime} A X \text {. }
$$

Now, the result of Proposition 5.1 follows from the lemma below (its proof follows from standard arguments on the concentration of $\chi^{2}$ variables, see e.g. Proposition 3 in Com01] and Lemma 1 in [LM00]):

Lemma 5.2 Let $X \in \mathbb{R}^{p}$ be a centered Gaussian vector with covariance matrix the identity. Let $\Gamma$ be a $p \times p$ Hermitian matrix (with complex entries). Let $\gamma_{1}, \ldots, \gamma_{p}$ be the eigenvalues of $\Gamma$. Define

$$
\gamma=\max _{1 \leq i \leq p}\left\{\left|\gamma_{i}\right|\right\} \text { and } s^{2}=\sum_{i=1}^{p}\left|\gamma_{i}\right|^{2}
$$

Then, for any $\eta>0$ one has that

$$
\mathbb{P}\left(\left|X^{\prime} \Gamma X-\operatorname{tr}(\Gamma)\right| \geq 2 \gamma \eta+2 \sqrt{s^{2} \eta}\right) \leq 2 \exp (-\eta),
$$

where $\operatorname{tr}(\Gamma)$ is the trace of the matrix $\Gamma$.

Then, remark that the eigenvalues of the $2 T \times 2 T$ Hermitian matrix $A_{x y}$ are smallest than $\frac{\rho_{x} \rho_{y}}{2 n}\|a\|^{2}$, where $\|z\|$ denotes the standard Euclidean norm of a vector $z$ in $\mathbb{C}^{T}$. Therefore, if one denotes by $\gamma_{1}, \ldots, \gamma_{p}$ the eigenvalues of $A$ with $p=2 n T$, it follows that

$$
\max _{1 \leq i \leq p}\left\{\left|\gamma_{i}\right|\right\} \leq \frac{\rho_{x} \rho_{y}}{2 n}\|a\|^{2}
$$

Remark also that $A_{x y}^{2}$ is of rank 2 with eigenvalues bounded by $\left(\frac{\rho_{x} \rho_{y}}{2 n}\|a\|^{2}\right)^{2}$ and therefore

$$
\sum_{i=1}^{p}\left|\gamma_{i}\right|^{2}=\operatorname{tr}\left(A^{2}\right)=n \operatorname{tr}\left(A_{x y}^{2}\right) \leq 2 n\left(\frac{\rho_{x} \rho_{y}}{2 n}\|a\|^{2}\right)^{2}=\frac{\rho_{x}^{2} \rho_{y}^{2}}{2 n}\|a\|^{4} .
$$

Finally, note that

$$
\|a\|^{2}=\left\|\psi_{\omega, u}\right\|^{2} \text { where }\left\|\psi_{\omega, u}\right\|^{2}=\sum_{k=1}^{T}\left|\psi_{\omega, u}\left(t_{k}\right)\right|^{2} .
$$

Therefore, using that $\operatorname{tr}(A)=0$ and by applying Lemma 5.2 with $p=2 n T, \Gamma=A, \gamma=\frac{1}{2 n} \rho_{x} \rho_{y}\left\|\psi_{\omega, u}\right\|^{2}$ and $s^{2}=\frac{1}{2 n} \rho_{x}^{2} \rho_{y}^{2}\left\|\psi_{\omega, u}\right\|^{4}$, it follows that for any $\eta>0$

$$
\mathbb{P}\left(|Z| \geq \frac{\rho_{x} \rho_{y}}{n}\left\|\psi_{\omega, u}\right\|^{2}(\eta+\sqrt{2 n \eta})\right) \leq 2 \exp (-\eta)
$$

Thus, the result of Proposition 5.1 follows by taking $\eta=-\log (\alpha / 2)$ which completes the proof. 


\section{References}

[AM10] D.P. Allen and C. D. MacKinnon. Time-frequency analysis of movement-related spectral power in eeg during repetitive movements: a comparison of methods. Journal of Neuroscience Methods, 186(1):107-115, 2010.

[BD04] G. Buzsaki and A. Draguhn. Neuronal oscillations in cortical networks. Science, 304:1926-1929, 2004.

[BL08] P.J. Bickel and E. Levina. Covariance regularization by thresholding. Ann. Statist., 36(6):2577-2604, 2008.

[CAvSRA01] Ombao H. C, Raz J. A, von Sachs R., and Malow B. A. Automatic statistical analysis of bivariate nonstationary time series. Journal of the American Statistical Association, 96:543-560, June 2001.

$\left[\mathrm{CHF}^{+} 95\right]$ B.A. Conway, D.M. Halliday, S.F. Farmer, U. Shahani, P. Maas, and et al. Weir, AI. Synchronization between motor cortex and spinal motoneuronal pool during the performance of a maintained motor task in man. J. Physiol., 489(3):917-924, 1995.

[Com01] F. Comte. Adaptative estimation of the spectrum of a stationary gaussian sequence. Bernoulli, 7(2):267-298, 2001.

[DS01] K. R. Davidson and S. J. Szarek. Local operator theory, random matrices and Banach spaces. In Handbook of the geometry of Banach spaces, Vol. I, pages 317-366. NorthHolland, Amsterdam, 2001.

[EK07] N. El Karoui. Tracy-Widom limit for the largest eigenvalue of a large class of complex sample covariance matrices. Ann. Probab., 35(2):663-714, 2007.

[FAKF00] B. Feige, A. Aertsen, and R. Kristeva-Feige. Dynamic synchronization between multiple cortical motor areas and muscle activity in phasic voluntary movements. $J$. Neurophysiol., 84:2622-2629, 2000.

[GC88] H. Gish and D. Cochran. Generalized coherence. In International Conference on Acoustics, Speech, and Signal Processing, pages 2745-2748, 1988.

[GCB02] P. Grosse, M. Cassidy, and P. Brown. Eeg-emg, meg-emg and emg-emg frequency analysis: physiological principles and clinical applications. Clin. Neurophysiol., 113:15231531, 2002.

[GKK03] G. Gurley, T. Kijewski, and A. Kareem. First-and higher-order correlation detection using wavelet transforms. Journal of Neuroscience Methods, 129:188-201, 2003.

[GMJ04] A. Grinsted, J. C. Moore, and S. Jevrejeva. Application of the cross wavelet transform and wavelet coherence to geophysical time series. Nonlinear Processes in Geophysics, 11(5/6):561-566, 2004.

[HCFR98] D.M. Halliday, B.A. Conway, S.F. Farmer, and J.R. Rosenberg. Using electroencephalography to study functional coupling between cortical activity and electromyograms during voluntary contractions in humans. Neuroscience Letters, 241:5-8, 1998. 
[HFDKR00] H.J. Hermens, B. Freriks, C. Disselhorst-Klug, and G. Rau. Development of recommendations for semg sensors and sensor placement procedures. J. Electromyogr Kinesiol., 10(5):361-374, 2000.

$\left[\mathrm{HRA}^{+} 95\right] \quad$ D. M. Halliday, J. R. Rosenberg, A. M. Amjad, P. Breeze, B. A. Conway, and S. F. Farmer. A framework for the analysis of mixed time series/point process data - theory and application to the study of physiological tremor, single motor unit discharges and electromyograms. Progr. Biophys. Mol. Biol., 64(2/3):237-278, 1995.

$\left[\mathrm{KBS}^{+} 00\right] \quad$ J.M. Kilner, S.N. Baker, S. Salenius, R. Hari, and R.N. Lemon. Human cortical muscle coherence is directly related to specific motor parameters. J. Neurosci., 20:8838-8845, 2000 .

$\left[\mathrm{LLR}^{+} 02\right] \quad$ J. P. Lachaux, A. Lutz, D. Rudrauf, D. Cosmelli, M. Le Van Quyen, J. Martinerie, and F. Varela. Estimating the time-course of coherence between single-trial brain signals: an introduction to wavelet coherence. Neurophysiol Clin, 32(3):157-174, June 2002.

[LM00] B. Laurent and P. Massart. Adaptive estimation of a quadratic functional by model selection. Annals of Statistics, 28:1302-1338, 2000.

[Mal98] S. Mallat. A Wavelet Tour of Signal Processing. Academic Press, New York, 1998.

[MBS01] J.F. Marsden, P. Brown, and S. Salenius. Involvement of the sensorimotor cortex in physiological force and action tremor. Neuroreport, 12:1937-1941, 2001.

[MH99a] T. Mima and M. Hallett. Corticomuscular coherence: a review. J. Clin. Neurophysiol., 16:501-511, 1999.

[MH99b] T. Mima and M. Hallett. Electroencephalographic analysis of cortico-muscular coherence: reference effect, conduction and generator. Clin. Neurophysiol., 110:1892-1899, 1999.

[MK04] D. Maraun and J. Kurths. Cross wavelet analysis: significance testing and pitfalls. Nonlinear Processes In Geophysics, 11:505-514, 2004.

[MN08] Y. Masakado and J.B. Nielsen. Task-and phase-related changes in cortico-muscular coherence. Keio J Med., 57(1):50-56, 2008.

[MSF07] E. Maris, J-M. Schoffelen, and P. Fries. Nonparametric statistical testing of coherence differences. Journal of Neuroscience Methods, 163(1):161-175, June 2007.

[MSGH00] T. Mima, J. Steger, C. Gerlo, and M. Hallett. Electroencephalographic measurement of motor cortex control of muscle activity in humans. Clin. Neurophysiol., 111:326-337, 2000 .

[OVB08] Hernando Ombao and Sébastien Van Bellegem. Evolutionary coherence of nonstationary signals. IEEE Trans. Signal Process., 56(6):2259-2266, 2008.

[PLJN06] M.A. Perez, J. Lundbye-Jensen, and J.B. Nielsen. Changes in corticospinal drive to spinal motoneurones following visuo-motor skill learning in humans. J. Physiol., 573(3):843-855, 2006. 
$\left[\mathrm{RGM}^{+} 09\right]$ J. Raethjen, R.B. Govindan, M. Muthuraman, F. Kopper, J. Volkmann, and G. Deuschl. Cortical correlates of the basic and first harmonic frequency of parkinsonian tremor. Clin. Neurophysiol., 120:1866-1872, 2009.

[SFJ10] J. Sanderson, P. Fryzlewicz, and M. W. Jones. Estimating linear dependence between nonstationary time series using the locally stationary wavelet model. Biometrika, 97(2):435-446, 2010.

[SG05] A. Schnitzler and J. Gross. Normal and pathological oscillatory communication in the brain. Nature Reviews Neuroscience, 6:285-296, 2005.

[SH03] S. Salenius and R. Hari. Synchronous cortical oscillatory activity during motor action. Current opinion in neurobiology, 13(6):678-684, 2003.

$\left[\mathrm{SPK}^{+} 97\right] \quad$ S. Salenius, K. Portin, M. Kajola, R. Salmelin, and R. Hari. Cortical control of human motoneuron firing during isometric contraction. J. Neurophysiol., 77:3401-3405, 1997.

[TBBDP96] C. Tallon-Baudry, O. Bertrand, C. Delpuech, and J. Pernier. Stimulus specificity of phase-locked and non-phase-locked 40hz visual responses in human. J. Neurosci., 16(13):4240-4249, July 1996.

[VLRM01] F. Varela, J-P. Lachaux, E. Rodriguez, and J. Martinerie. The brainweb: Phase synchronization and large-scale integration. Nature Reviews Neuroscience, 2(4):229239, April 2001.

[WCB05] Brandon Whitcher, Peter F. Craigmile, and Peter Brown. Time-varying spectral analysis in neurophysiological time series using hilbert wavelet pairs. Signal Process., 85:2065-2081, November 2005.

[Whi00] Brandon Whitcher. Wavelet analysis of covariance with application to atmospheric time series. J. Geophys. Res, 105:200-0, 2000.

$\left[\mathrm{ZHJ}^{+}\right.$06] Y. Zhan, D. Halliday, P. Jiang, X. Liu, and J. Feng. Detecting the time-dependent coherence between non-stationary electrophysiological signals-a combined statistical and time-frequency approach. Journal of Neuroscience Methods, 156:322-332, 2006. 